YOUNG STELLAR OBJECTS AND TRIGGERED STAR FORMATION IN THE VULPECULA OB ASSOCIATION

This article has been downloaded from IOPscience. Please scroll down to see the full text article.

2010 ApJ 712797

(http://iopscience.iop.org/0004-637X/712/2/797)

The Table of Contents and more related content is available

Download details:

IP Address: 131.215.193.213

The article was downloaded on 26/03/2010 at 21:29

Please note that terms and conditions apply. 


\title{
YOUNG STELLAR OBJECTS AND TRIGGERED STAR FORMATION IN THE VULPECULA OB ASSOCIATION
}

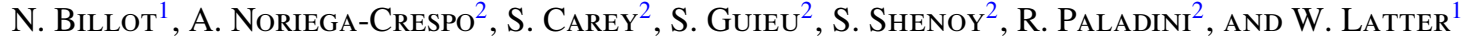 \\ ${ }^{1}$ NASA Herschel Science Center, IPAC, MS 100-22, California Institute of Technology, Pasadena, CA 91125, USA; nbillot@ipac.caltech.edu \\ ${ }^{2}$ Spitzer Science Center, IPAC, MS 220-6, California Institute of Technology, Pasadena, CA 91125, USA \\ Received 2009 November 16; accepted 2010 February 5; published 2010 March 8
}

\begin{abstract}
The Vulpecula OB association, VulOB1, is a region of active star formation located in the Galactic plane at $2.3 \mathrm{kpc}$ from the Sun. Previous studies suggest that sequential star formation is propagating along this $100 \mathrm{pc}$ long molecular complex. In this paper, we use Spitzer MIPSGAL and GLIMPSE data to reconstruct the star formation history of Vul OB1, and search for signatures of past triggering events. We make a census of young stellar objects (YSOs) in Vul OB1 based on IR color and magnitude criteria, and we rely on the properties and nature of these YSOs to trace recent episodes of massive star formation. We find 856 YSO candidates, and show that the evolutionary stage of the YSO population in VulOB1 is rather homogeneous-ruling out the scenario of propagating star formation. We estimate the current star formation efficiency to be $\sim 8 \%$. We also report the discovery of a dozen pillar-like structures, which are confirmed to be sites of small scale triggered star formation.
\end{abstract}

Key words: H II regions - infrared: ISM - infrared: stars - stars: formation - stars: pre-main sequence

Online-only material: color figures, machine-readable table

\section{INTRODUCTION}

The physical mechanisms describing stellar birth are fairly well understood for low- and intermediate-mass stars but still under debate for their high-mass analogs (McKee \& Ostriker 2007). On larger scales, observations show that star-forming mechanisms are of relatively poor efficiency, as only a fraction of the gas reservoir in the universe is turning into stars. Typical star formation efficiencies (SFEs) are of the order 3\%-6\% in our Galaxy (Evans et al. 2009), and 5\% or less in other galaxies (Rownd \& Young 1999). Still, in extreme environments like in the starburst galaxy Arp 220, the SFE can reach 50\% (Anantharamaiah et al. 2000), suggesting that the star-forming mechanism at work in such objects could be of a different nature. For instance, the feedback into the interstellar medium (ISM) from short-lived massive stars seems to influence the yield of star formation in their local environment. As they evolve off the main sequence, high-mass stars produce a copious amount of energy while still embedded in their native cocoon; such disruption of a molecular cloud leads to gravitational instabilities and possibly to the onset of a new episode of star formation. Hosokawa \& Inutsuka (2006) showed that under certain conditions runaway triggering can take place around massive OB stars. In other cases however, turbulence and magnetic fields can have a negative feedback on the local ISM and lead to the suppression of the star-forming activity (Price \& Bate 2009; Stone 1970). Elmegreen (1998) provides an exhaustive review of the theoretical framework to study the dynamical triggers of star formation.

In this context, we use the data from the Spitzer Legacy surveys MIPSGAL (Carey et al. 2009) and GLIMPSE (Benjamin et al. 2003) to study the star-forming activity currently taking place in the Vulpecula OB association (hereafter Vul OB1). Vul OB1 hosts nearly one hundred OB stars and three bright H II regions known as Sharpless objects 86, 87, and 88 (Sharpless 1959). According to Ehlerová et al. (2001) and Turner (1986), the star-forming activity occurring in Vul OB1 might have been triggered by a common external source, and star formation might be propagating from one $\mathrm{H}$ II region to another through the expansion of a supernova shock front. The aim of this paper is to study the triggered star formation on scales as large as $\sim 100$ pc in VulOB1, which could help us understand star formation mechanisms within other giant molecular clouds in the Galaxy and beyond.

We use the method developed by Gutermuth et al. (2008) to obtain a census of young stellar objects (hereafter YSOs) in Vul OB1 based on infrared color and magnitude criteria, and we rely on the properties and nature of these YSOs to trace episodes of star formation. The MIPSGAL and GLIMPSE sensitivity makes our search for YSOs in Vul OB1 biased toward massive objects that reveal the most recent star-forming activity.

In Section 2, we present the data set used in the paper. In Section 3, we give a comprehensive description of Vul OB1 encompassing the three $\mathrm{H}$ II regions as well as the dozen pillarlike structures we discovered in this region. Section 4 describes the identification process of YSOs based on their IR-excess emission. We present and discuss our results in Section 5, and we give our conclusions in Section 6.

\section{THE DATA SET}

\subsection{Spitzer Observations and Point Source Catalogs}

The Spitzer Space Telescope (Werner et al. 2004) observed over $270 \mathrm{deg}^{2}$ of the inner Galactic plane in six wavelength bands as part of two legacy programs: the Galactic Legacy Infrared Mid-Plane Survey Extraordinaire (GLIMPSE; PI: E. Churchwell; Benjamin et al. 2003) and the MIPS GALactic plane survey (MIPSGAL; S. Carey PI; Carey et al. 2009). The Vulpecula region was covered by GLIMPSE I (PID 188) and MIPSGAL I (PID 20597). It was imaged with the Infrared Array Camera (IRAC; Fazio et al. 2004) at 3.6, 4.5, 5.8, and $8.0 \mu \mathrm{m}$, and with the MIPS camera (Rieke et al. 2004) at 24 and $70 \mu \mathrm{m}$. The angular resolution of Spitzer is $2^{\prime \prime}, 6^{\prime \prime}$, and $18^{\prime \prime}$ at 8, 24, and $70 \mu \mathrm{m}$, respectively.

The MOPEX package (Makovoz et al. 2006) was used to build mosaics of the sky about $1 \mathrm{deg}^{2}$ wide from individual Basic Calibrated Data (BCD) frames. Details of the post-processing and $\mathrm{BCD}$ pipeline modifications are described in Meade et al. 


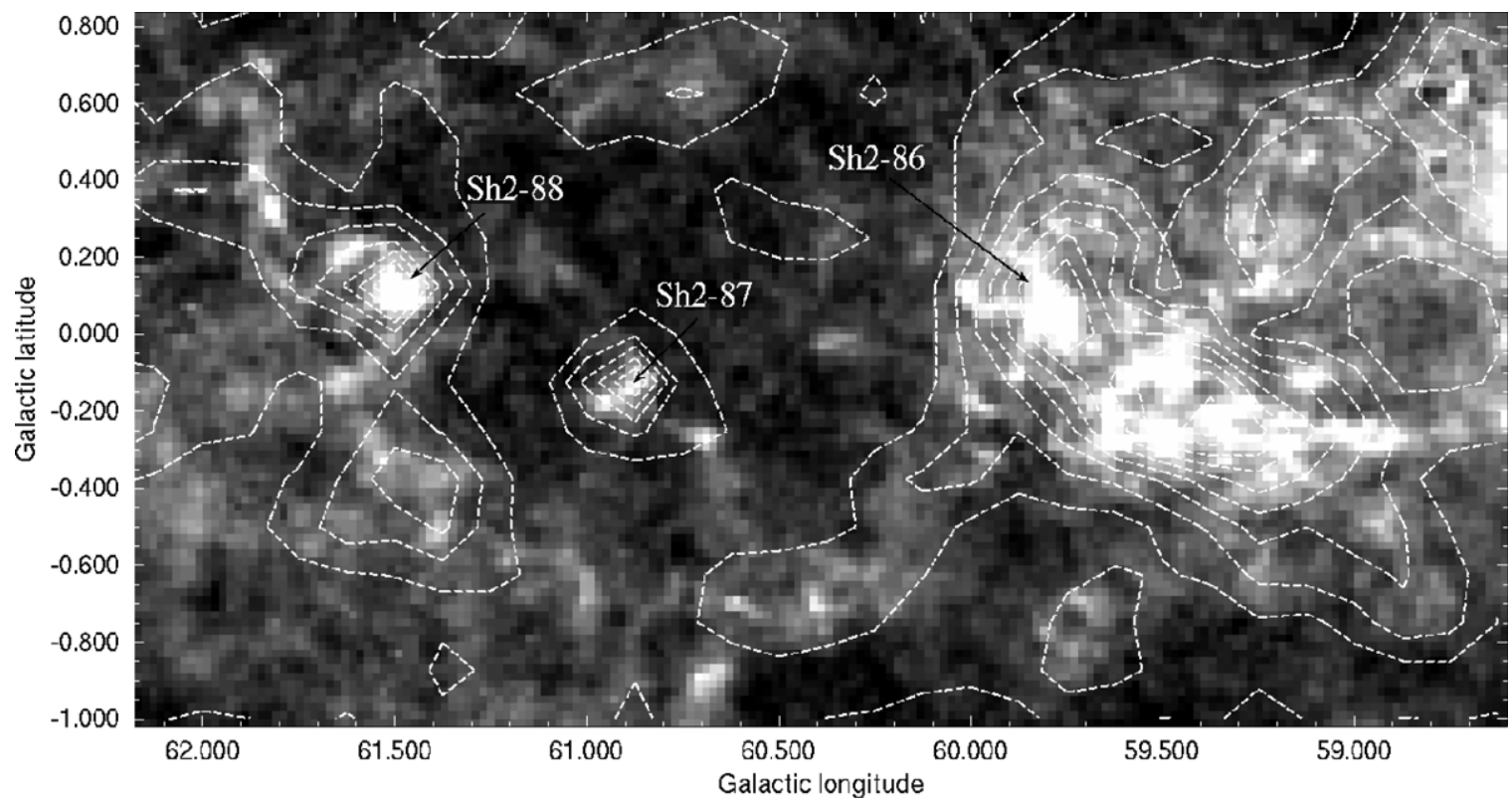

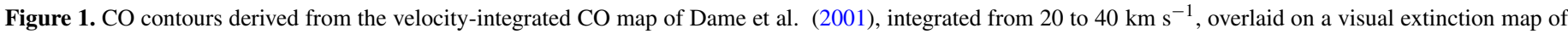
Vul OB1 (Av ranges from 3 to 20 mag on a linear scale). The Sharpless Objects appear as higher extinction regions.

(2007) for GLIMPSE and in Mizuno et al. (2008) for MIPSGAL. The large Vulpecula mosaics presented in this paper are also built with MOPEX using the $1 \mathrm{deg}^{2}$ plates available through the Infrared Science Archive ${ }^{3}$ (IRSA).

The search for YSO candidates in Vulpecula relies on the Point Source Catalogs (PSCs) generated from the GLIMPSE and MIPSGAL surveys. For GLIMPSE, point sources are extracted using a modified version of the point-spread function (PSF) fitting program DAOPHOT (Stetson 1987). The GLIMPSE PSC we used is the enhanced data product v2.0 available on the IRSA Web site. For MIPSGAL, sources are extracted from the $24 \mu \mathrm{m}$ maps using a Pixel-Response Function fitting method (S. Shenoy et al. 2010, in preparation) and the APEX software developed at the Spitzer Science Center. ${ }^{4}$ This catalog is then merged with the GLIMPSE PSC to include IRAC and Two Micron All Sky Survey (2MASS) $)^{5}$ fluxes if they are available. $70 \mu \mathrm{m}$ sources are extracted from reprocessed $70 \mu \mathrm{m}$ maps (R. Paladini et al. 2010, in preparation) using the StarFinder software (Diolaiti et al. 2000), and matched to the MIPSGAL PSC with a search radius of $9 "$.

\subsection{Ancillary Data}

We complement our data set with visible, submillimeter, millimeter, and radio data to obtain a broader view of the Vulpecula complex, and to investigate the morphology, star formation history, and possible relationship between the $\mathrm{H}$ II regions. We make use of the VLA Galactic Plane Survey (VGPS) data, which consists of $\mathrm{H}$ I line and $21 \mathrm{~cm}$ continuum emission data with a resolution of $1^{\prime} \times 1^{\prime} \times 1.56 \mathrm{~km} \mathrm{~s}^{-1}$ and $1^{\prime}$, respectively (Stil et al. 2006). These data are analyzed in details in Section 3.1. We also use the Virginia Tech Spectral-line

\footnotetext{
http://irsa.ipac.caltech.edu/data/SPITZER/GLIMPSE/ and http://irsa.ipac.caltech.edu/data/SPITZER/MIPSGAL/

4 http://ssc.spitzer.caltech.edu/dataanalysistools/tools/mopex

5 The 2MASS is a joint project of the University of Massachusetts and the Infrared Processing and Analysis Center/California Institute of Technology, funded by the National Aeronautics and Space Administration and the National Science Foundation.
}

Survey (VTSS) which provides arcminute-resolution images of the $6563 \AA \mathrm{H} \alpha$ recombination line of atomic hydrogen in the Vulpecula region (Dennison et al. 1998). S. Bontemps (2009, private communication; see Schneider et al. 2006 for details) also provided our team with a 1.2-resolution extinction map of VulOB1 generated from stellar counts and colors derived from the 2MASS PSC (see Figure 1). The visual extinction in Vulpecula ranges from 3 to 20 with a distribution peaking at Av $\sim 5$. We also use the CO survey of our Galaxy published in Dame et al. (2001). However, the relatively low spatial resolution of $\sim 7.5$ over Vul OB 1 prevented a relevant detailed study of the CO emission. We find nonetheless a very good correlation between the $\mathrm{CO}$ emission and the visual extinction that both trace the cold and dusty molecular material (cf. Figure 1). In addition, we used the submillimeter data obtained during the second flight of the Balloon-borne Large Aperture Submillimeter Telescope (BLAST; Pascale et al. 2008), where a $4 \mathrm{deg}^{2}$ region was mapped around Sh2-86 at 250, 350, and $500 \mu \mathrm{m}$ (Chapin et al. 2008). The angular resolution of the BLAST maps are $40^{\prime \prime}, 50^{\prime \prime}$, and $60^{\prime \prime}$ at 250,350, and $500 \mu \mathrm{m}$, respectively. And finally, we exploit the recently released $1.1 \mathrm{~mm}$ data of the Galactic Plane Survey carried out with Caltech Submillimeter Observatory/Bolocam (J. Aguirre et al. 2010, in preparation) and providing an angular resolution of $31^{\prime \prime}$.

\section{THE VULPECULA OB ASSOCIATION}

VulOB1 is an active star-forming region located in the Galactic plane at a longitude $1 \sim 60^{\circ}$. In this article, we focus on the region centered at $(l, b)=(60.2,-0.2)$ and covering about $6.6 \mathrm{deg}^{2}$ (see Figures 1 and 2). Garmany \& Stencel (1992) describe Vul OB1 as an oval region about 3.5 by 1.5 hosting the star cluster NGC 6823. Nearly one hundred hot massive stars are found in the direction of Vulpecula from the catalog of OB stars compiled by Reed (2003). Some of these stars have already been associated with and are responsible for the ionization of three bright $\mathrm{H}$ II regions in Vul OB1, namely Sh2-86, 87, and 88 (Sharpless 1959). Figure 2 presents the midinfrared morphology of VulOB1 and the location of the OB stars. 

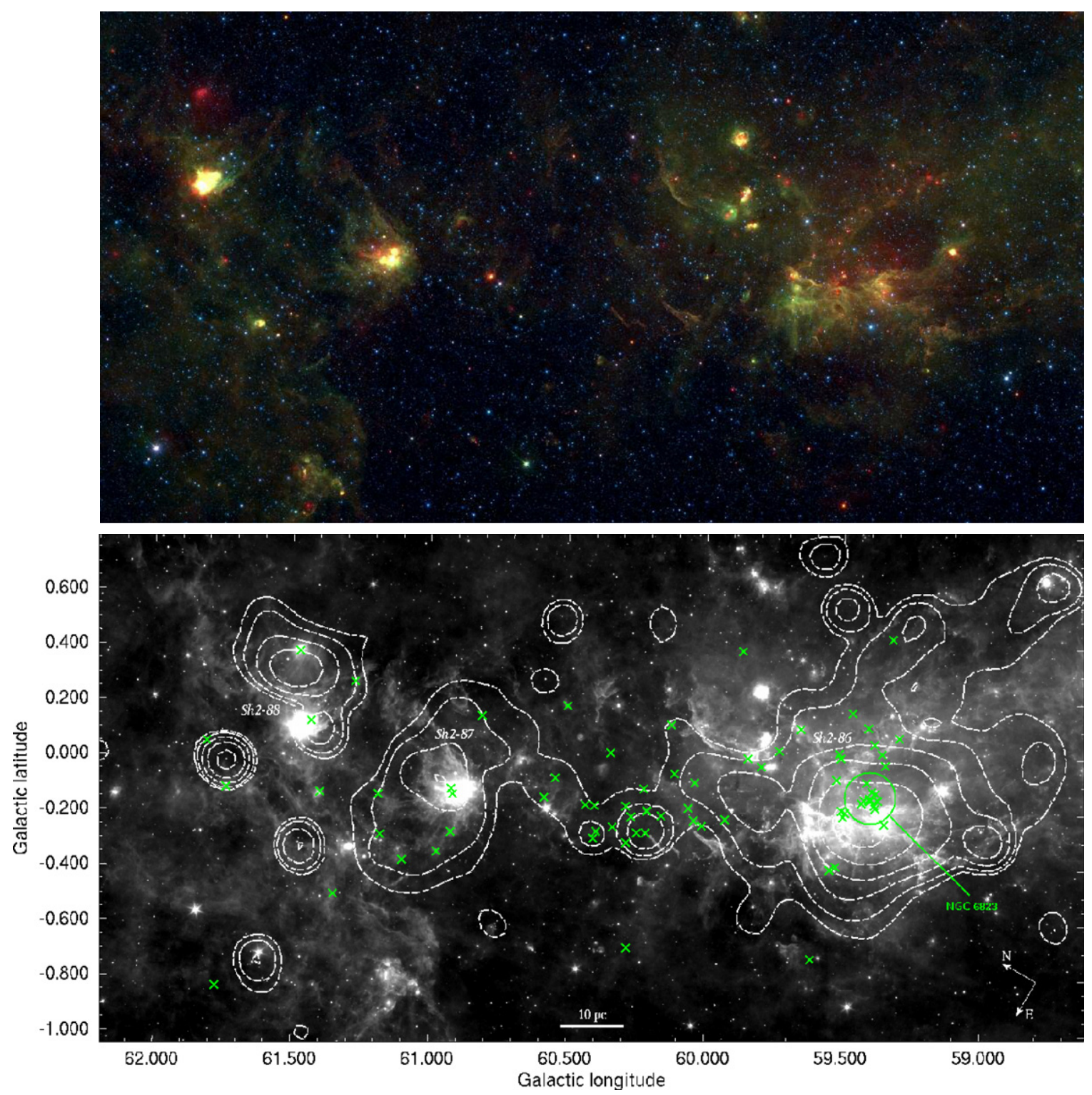

Figure 2. Top panel shows composite image of VulOB1 (3.6, 8.0, and $24 \mu \mathrm{m}$ are color coded as blue, green, and red, respectively). This image was assembled by $\mathrm{R}$. Hurt and T. Robitaille based on GLIMPSE and MIPSGAL data. The bottom panel represents H $\alpha$ contours from the VTSS overlaid on the MIPSGAL $24 \mu \mathrm{m}$ image. The $\mathrm{H} \alpha$ emission associated with Sh2-88 peaks at $(l, b)=(61.45,0.30)$ while the bright mid-IR emission located $15^{\prime}$ southeast of the diffuse nebula is the knot identified in the text as Sh2-88B. Green crosses indicate the location of the $88 \mathrm{OB}$ stars present in this $3.7 \times 1.8$ region.

The VGPS $21 \mathrm{~cm}$ continuum emission covering VulOB1 reveals two bright circular compact sources $(\sim 1$ '.3 in diameter) coincident with Sh2-87 and Sh2-88, and an extended dimmer region $\left(27^{\prime} \times 12^{\prime}\right)$ reminiscent of the infrared morphology of Sh2-86. The supernova remnant SNR G59.5+0.1 (Taylor et al. 1992) is also clearly visible in the radio continuum data at $(l, b)=(59.59,0.1)$, its measured diameter is $15^{\prime}$ (cf. Section 5.4.1). Our analysis of the VGPS H I line data indicates that the three H II regions are neighbors (cf. Section 3.1), as was previously noted by Turner (1986), Ehlerová et al. (2001), and Cappa et al. (2002). Distance determinations for the three Sharpless objects and their exciting stars range from 1.5 to 3.2 kpc (Fich \& Blitz 1984; Barsony 1989; Guetter 1992; Brand \& Blitz 1993; Massey et al. 1995; Deharveng et al. 2000; Hoyle et al. 2003; Kharchenko et al. 2005; Bica et al. 2008). Following the arguments from Chapin et al. (2008), we adopt a common distance of $2.3 \mathrm{kpc}$ for the three $\mathrm{H}$ II regions.
In the catalog of Reed (2003) we find about 30 OB stars in the direction of Sh2-86 and Sh2-87, in what we identify as the pillars region (these stars might be responsible for the existence of pillar structures; see Section 3.2). We check their possible association with Vul OB1 by computing their distance moduli based on the $M_{V}$-spectral type relation and the intrinsic colors given by Aller et al. (1982). We assume a ratio of total-toselective absorption $R_{\mathrm{V}}=3.1$ typical of the diffuse ISM, and we associate a visual extinction to each star based on our extinction map of VulOB1. Out of the 16 stars for which spectral type and magnitude information are available in the Reed catalog, 12 fall between 1.3 and $2.3 \mathrm{kpc}$ with a mean distance from the Sun of $1.91 \pm 0.4 \mathrm{kpc}$. We find similar distances using intrinsic colors and absolute magnitudes given by Johnson (1958) and Wegner (2006), respectively. Although the uncertainties are significant, which is mostly due to the large uncertainty on the values of the absolute magnitudes, our distance estimate for 
these stars is consistent with an association with the Vulpecula complex.

\subsection{H II Regions}

Sh2-86 is an extended H II region about 40' wide (Sharpless 1959) located in the southern part of Vulpecula (Figures 1 and 2 and Figure 11 in the Appendix). It is excited by the open cluster NGC 6823, which is part of the OB association Vul OB1 (Massey et al. 1995; Bica et al. 2008). Pigulski et al. (2000) estimate the age of the star cluster at $3 \pm 1$ Myr old. In the atomic gas distribution derived from the VGPS data, Sh2-86 appears as an oblong hole at velocities between 26 and $31 \mathrm{~km} \mathrm{~s}^{-1}$. We find that the dimension of the shell is approximately $60^{\prime}$ along the Galactic plane and $25^{\prime}$ across it. In the optical, the southeastern part of Sh2-86 is very contrasted. It exhibits silhouetted pillar-like structures pointing toward NGC 6823, emission and reflection nebulae as well as filamentary structures. The infrared emission is also contrasted in this region tracing the complex interplay between the UV radiation and the surrounding dusty ISM.

Chapin et al. (2008) also report the detection of 49 compact submillimeter sources associated with Sh2-86 using BLAST observations. The presence of these clumps, with masses ranging from 14 to $700 M_{\odot}$, indicates the capability of Sh2-86 to form massive stars.

Sh2-87 and Sh2-88 are also H II regions initially discovered by Sharpless (1959). These regions are active sites of star formation as indicated by the presence of $\mathrm{H}_{2} \mathrm{O}$ maser line emissions and bipolar outflows (Barsony 1989; Deharveng et al. 2000). Bolocam data show bright sources at the location of Sh2-87 and Sh2-88 indicating the presence of cold core candidates. Our analysis of the VGPS data reveals that the morphology of the Hi shell around Sh2-87 at velocities between 22 and $26 \mathrm{~km} \mathrm{~s}^{-1}$ is very similar to that of the $24 \mu \mathrm{m}$ emission. The H II region appears as a hole in the $\mathrm{H} \mathrm{I}$ distribution as the hydrogen is ionized by an embedded B0 star (Felli \& Harten 1981). A compact source seen in absorption sits at the center of Sh2-87, its position is coincident with the location of the peak emission at $24 \mu \mathrm{m}(l, b)=(60.88,-0.13)$. Barsony (1989) and Xue \& $\mathrm{Wu}$ (2008) give a detailed description of this $\mathrm{H}$ II region.

Sh2-88 is a diffuse nebula of diameter $\sim 20^{\prime}$ located at $(l, b)=(61.45,0.34)$, which is excited by the $\mathrm{O} 8 \mathrm{star}$ $\mathrm{BD}+25^{\circ} 3952$ (Cappa et al. 2002). The star formation activity taking place in Sh2-88 occurs in a couple of nebular knots identified by Lortet-Zuckermann (1974), Sh2-88A and Sh2-88B, located $15^{\prime}$ southeast of Sh2-88. These knots are responsible for the bright $24 \mu \mathrm{m}$ emission visible in Figure 2 at $(l, b)=(61.47,0.1)$. Sh2-88B is actually the brightest of the three Sharpless objects at mid-IR wavelengths. Figure 10 in the Appendix shows a composite image of Sh-88B in IRAC bands. It consists of a compact cometary $\mathrm{H}$ II region and an ultracompact (UC) H in region. Deharveng et al. (2000) present a detailed analysis of Sh2-88B and its stellar content. At radio wavelength, the association of Sh2-88 with a H I shell is somewhat more difficult than in the other two cases as the edges of the shell are not as well defined. We detect, however, a faint hole at velocities between 22 and $26 \mathrm{~km} \mathrm{~s}^{-1}$ as well as a compact source seen in absorption in the center of Sh2-88B.

\subsection{Pillar Structures}

We have discovered several pillar-like structures on either side of the Galactic equator between Sh2-86 and Sh2-87 $\left(59.5<l<61^{\circ}\right)$. These objects are similar to the archetypal pillars of creation found in M16 (Hester et al. 1996; Urquhart et al. 2003). Such pillars are usually associated with recent episodes of star formation as the winds and radiation emanating from young massive stars are responsible for sculpting these elongated elephant trunks out of the surrounding molecular material. An accepted mechanism for the formation of the pillars is the slow photoevaporation of a pre-existing molecular clump shadowing a tail of more diffuse gas, but other mechanisms have been proposed based on hydrodynamical instabilities for instance. Spitzer (1954), Bertoldi (1989), Lefloch \& Lazareff (1994), and Carlqvist et al. (2003) present analytical models for the formation of pillar-like structures, and Miao et al. (2006), Mizuta et al. (2006), and Gritschneder et al. (2009) carry out numerical simulations of the formation and evolution of such objects. According to these studies, the pressure at the surface of the pillars due to the strong external radiation appears to trigger the formation of new stars, which is confirmed by recent observations (e.g., Sugitani et al. 2002; Reach et al. 2004, 2009; Bowler et al. 2009).

Figure 3 presents the $24 \mu \mathrm{m}$ image of the pillars found in Vulpecula, and gives the nomenclature to identify individual objects. The three pillars VulP12-13-14 were already known prior to these observations (Chapin et al. 2008); they are located northeast of NCG 6823 and are obviously associated with NGC 6823 as they point toward the star cluster. However, the 11 pillars VulPl to VulPll have never been reported in the literature before this study. Their association with VulOB1 is not as straightforward as in the case of NGC 6823 since no obvious source could be identified as their sculptor. The OB stars mentioned previously are certainly good candidates, for instance, the star HD 186746 (spectral type B8Ia) is located right above the tip of the pillar $V u l P l$ in projection (Figures 2 and 3). Nevertheless, 10 of the discovered pillars, out of 11 , seem to point toward the same faint diffuse nebulosity located at $(l, b)=(60.37,-0.04)$, which is not coincident with any known OB stars. We argue that the chance for 10 randomly distributed pillars to point toward the same object is negligible ${ }^{6}$ thus the discovered pillars VulP1-VulP10 are most certainly associated with each other. The central nebulosity might host the object responsible for the formation of the pillars. In addition, a diffuse faint circularly symmetric structure centered on the same nebulosity is discernible in the background diffuse emission. Bright objects at $24 \mu \mathrm{m}$ also seem to delineate this faint structure at a radius of $\sim 15^{\prime}$ centered at $(l, b)=(60.37,-0.04)$. These morphological features suggest that a single event in the past may have molded the pillars region.

We analyze the radio data in the pillars region to look for a possible association between these structures and the OB association. Out of the 15 pillars identified in Figure 3, only three are detected in the VGPS H I line data. The pillar VulP10 presents a deep absorption feature at $27-33 \mathrm{~km} \mathrm{~s}^{-1}$ as well as smaller features centered at 8,13 , and $20 \mathrm{~km} \mathrm{~s}^{-1}$. These $\mathrm{H} \mathrm{I}$ features are marginally resolved into two close compact sources located at the base of the pillar. Since these features are quite different from the IR morphology of VulP10, we cannot exclude a fortuitous association with a coincident radio source seen in projection.

VulP4 and VulP5 appear to have similar characteristics; they present comparable morphologies in the $\mathrm{H}$ I line data and in the

\footnotetext{
6 Assuming we measure the direction of the pillars with an accuracy of $\sim 2^{\circ}$, the probability is lower than $10^{-11}$.
} 


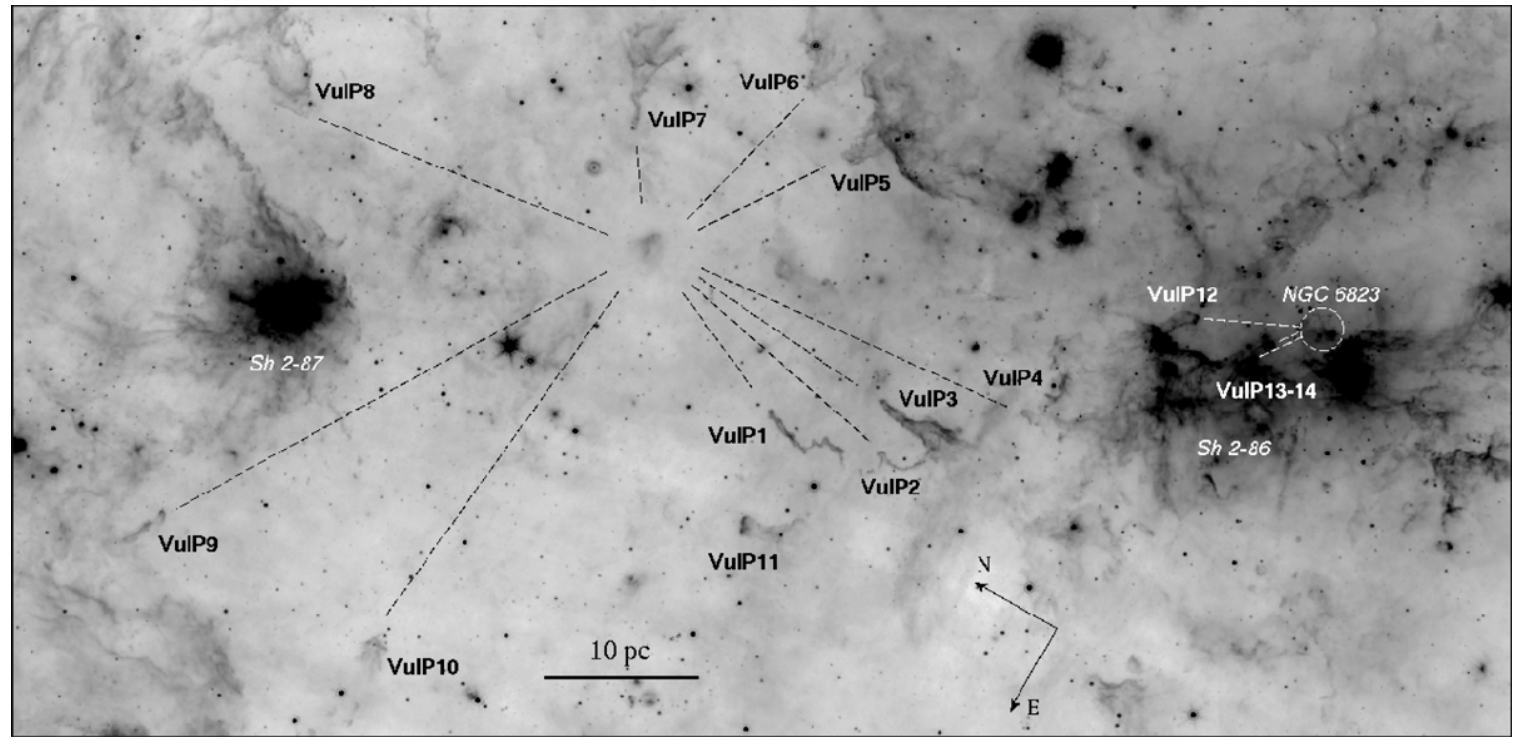

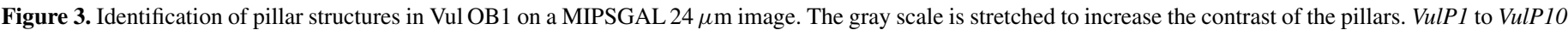

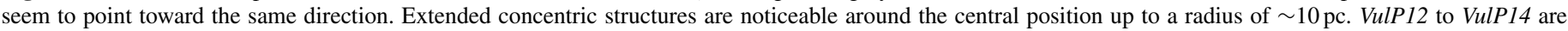
associated with the young star cluster NGC 6823.

Spitzer bands. They both emerge from the molecular cloud to be exposed to the ionizing radiation. These pillars are located at the edge of $\mathrm{H}$ I shells, and their silhouette is clearly identified at velocities $21-23 \mathrm{~km} \mathrm{~s}^{-1}$, which places them at roughly the same distance as the three Sharpless objects for which $V_{\mathrm{LSR}}=$ $22-31 \mathrm{~km} \mathrm{~s}^{-1}$.

VGPS continuum emission shows a bright two-lobed compact source located at the base of the pillar VulP10, consistent with the two blobs observed in the H I line data. No other pillars are detected in the $21 \mathrm{~cm}$ continuum.

Tables 3 and 4 in the Appendix summarize the geometrical and morphological information extracted from the Spitzer observations for individual pillars. Of particular interest are the bright ( $\sim 2 \mathrm{Jy}$ ) compact sources found at $70 \mu \mathrm{m}$ in the core of the pillars VulP1 and VulP3 (see Figure 4). These embedded sources are faint at $24 \mu \mathrm{m}(\sim 1 \mathrm{mJy})$ and have no counterparts in IRAC and 2MASS bands. Even though star formation is expected to occur preferentially at the tip of such structures, these red sources are likely sites of massive star formation. Longer wavelength ${ }^{7}$ observations would be necessary to identify the nature of these sources. Figure 4 shows the pillars VulP5 and VulP6 and their associated red sources. YSO candidates are located at the tip of each pillar, and also in the pedestal of VulP5 along some infrared dark clouds (IRDCs). Submillimeter BLAST sources are also located at the tip of the pillars and along the same IRDCs. Two Bolocam sources are also located along one of the IRDCs.

\section{THE IR-EXCESS EMISSION OF YOUNG STELLAR OBJECTS}

Stars form from the gravitational collapse and fragmentation of giant molecular clouds in the ISM. The contraction of the cold gas leads to the formation of a dense rotating core radiating its thermal energy in the millimeter and far-IR regime. As the bulk of the initial cloud mass is falling toward the center of the core, a protostar emerges with a gaseous and dusty accretion

\footnotetext{
7 The BLAST data do not cover these pillars, and the $1.1 \mathrm{~mm}$ Bolocam data, which is available for these pillars, is not as good a diagnostic as the submillimeter data.
}

disk rotating around it. The peak emission of such a YSO shifts toward shorter wavelengths, revealing a bright source in the midIR. The circumstellar disk is then dissipated via accretion, planet formation, or evaporation. Further reading on star formation mechanisms can be found in, e.g., Terebey et al. (1984), Adams et al. (1987), André et al. (1993, 2000), and McKee \& Ostriker (2007).

Recent works have shown that the wavelength coverage of Spitzer instruments is well suited for observing the midIR excess emission emanating from the warm circumstellar material around YSOs (e.g., Allen et al. 2004; Harvey et al. 2007; Koenig et al. 2008; Guieu et al. 2009). Based on the midIR extinction law (Lutz 1999; Indebetouw et al. 2005; Flaherty 2007), Gutermuth et al. (2008) furthermore argue that the most reliable IRAC-based criterion for identifying YSO candidates is the [4.5] - [5.8] color. Indeed, the flattening of the extinction curve observed between the IRAC bands 2 and $4(4.5$ to $8 \mu \mathrm{m})$ reduces the degeneracy between selective interstellar extinction and intrinsic IR excess.

Our approach to identify YSOs in VulOB1 is to exploit the method developed by Gutermuth et al. (2008) based on Spitzer colors and magnitude cuts to identify sources with IRexcess emission. We apply this method to the PSCs compiled by the GLIMPSE and MIPSGAL teams (Section 2.1) to take full advantage of the good photometric quality and the high reliability of these data. The direct consequence of this approach is that no extended sources will be considered in our study even though they exhibit significant IR-excess emission. To mitigate this caveat, we compare the angular resolution of Spitzer with the typical angular size of circumstellar envelops and disks seen at $2.3 \mathrm{kpc}$ from the Sun. Assuming the telescope optic is diffraction limited ${ }^{8}$ down to $5.5 \mu \mathrm{m}$, the resolution element at 3.6 and $24 \mu \mathrm{m}$ corresponds to 3500 and $16000 \mathrm{AU}$ at 2.3 $\mathrm{kpc}$, respectively. The spatial extent of the YSOs we are looking for does not exceed these limits. For instance, Vicente \& Alves (2005) measured the size distribution of circumstellar disks in the Trapezium cluster and found disk sizes ranging from 100

\footnotetext{
8 From the Spitzer Observer's Manual available at http://ssc.spitzer.caltech.edu.
} 

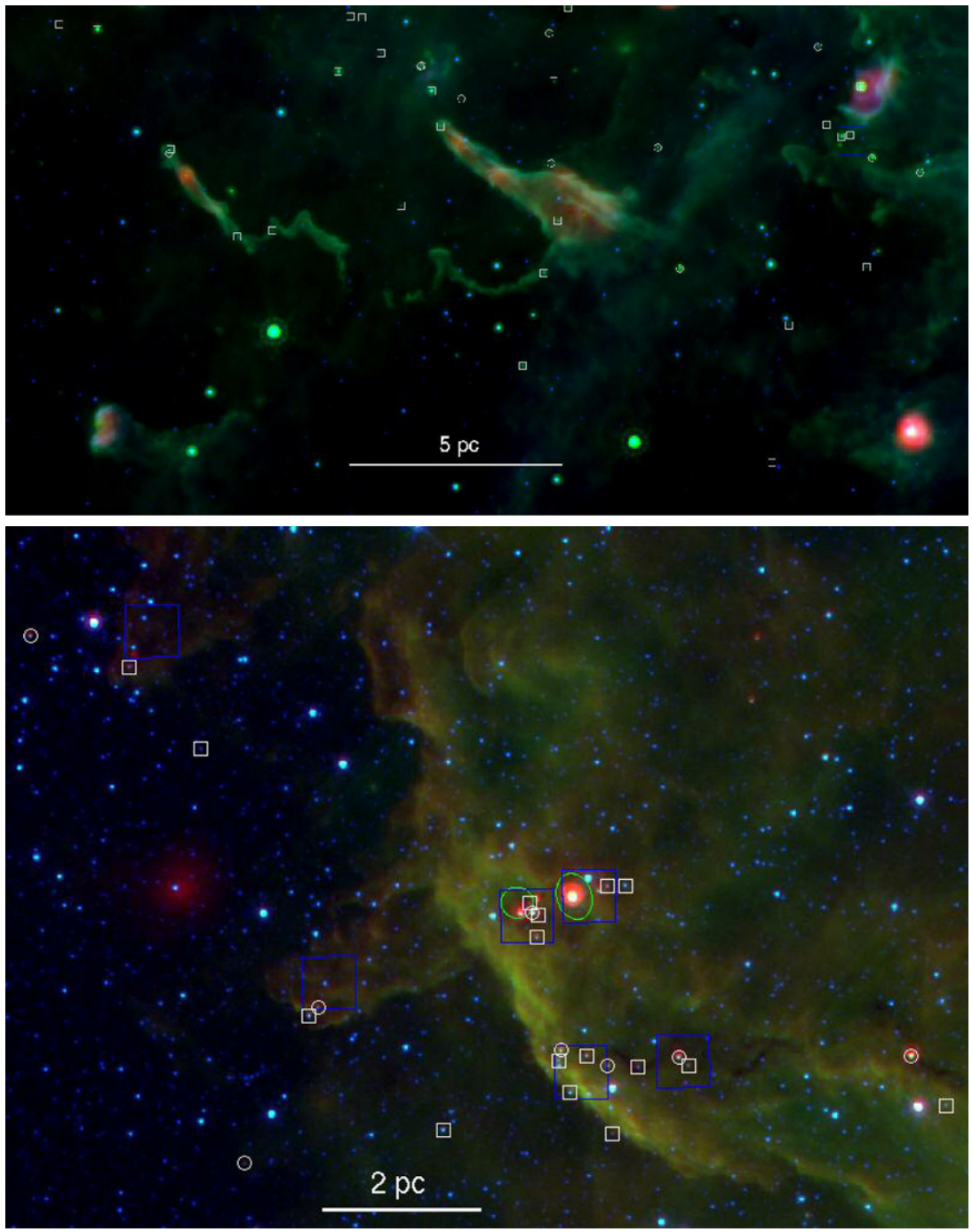

Figure 4. Spitzer three-color images of some pillars discovered in VulOB1. YSO candidates are marked as white small squares and circles, large blue boxes show BLAST sources, and green ellipses indicate Bolocam sources. Top panel: five pillars identified as VulP11 and VulP1 to VulP4 in Figure 3 (from left to right). Blue, green, and red are 8, 24, and $70 \mu \mathrm{m}$ images, respectively. VulP1 and VulP2 exhibit bright $70 \mu \mathrm{m}$ sources in their core. Bottom panel: VulP5 and VulP6. Blue, green, and red are respectively 4.5, 8.0, and $24 \mu \mathrm{m}$ images. Infrared dark clouds (IRDCs) are visible in the pedestal of the pillar VulP5. YSO candidates are aligned along IRDCs.

to $400 \mathrm{AU}$, an order of magnitude smaller than the resolution element at $3.6 \mu \mathrm{m}$. Although protostellar envelops have a larger extend, i.e., a few thousand $\mathrm{AU}$ according to Bonnell et al. (1996) and Jørgensen et al. (2002), and assuming they can only be detected at long wavelengths where the resolution element is larger, we expect the number of resolved YSOs in Vul OB1 to be relatively small. However a fraction of the YSOs in their early phases of evolution might be resolved by Spitzer, even at $24 \mu \mathrm{m}$, and those large protostars would be excluded from our analysis.

\subsection{Initial Catalog}

We first compile a catalog of IRAC point sources based on the highly reliable PSC available from the IRSA Web site as a GLIMPSE I v2.0 enhanced data product. ${ }^{9}$ All sources found in this catalog with galactic coordinates in the range $58.6<l<62.2$ and $-1.0<b<0.8$ are considered $\left(\sim 3.28 \times 10^{5}\right.$ sources). Fluxes are converted to magnitudes using the zero points given in Table 1 . To ensure good photometry

\footnotetext{
9 http://www.astro.wisc.edu/glimpse/glimpse1_dataprod_v2.0.pdf
}

and avoid flux stealing between adjacent sources as noted by Robitaille et al. (2008), we require that valid point sources have magnitude errors lower than $0.2 \mathrm{mag}$, and that their close source flag ${ }^{10}$ (csf) is zero. We also apply specific cuts on IRAC magnitudes to achieve high detection reliability; the magnitude limits we adopt are 14.2,14.1,11.9, and 10.8 mag at 3.6, 4.5, 5.8 , and $8.0 \mu \mathrm{m}$, respectively. According to the GLIMPSE I Assurance Quality Document, ${ }^{11}$ these limits ensure a detection reliability above $98 \%$ in all four IRAC bands. The reliability of the faintest sources however might vary across the Vul OB1 region as it depends on the background brightness structure and the local source density.

This intermediate catalog is then merged with the MIPSGAL $24 \mu \mathrm{m}$ PSC (S. Shenoy et al. 2010, in preparation) with the constraint that the maximum separation between two matching sources is $3^{\prime \prime}$. Once again to ensure a good photometry, we require that the magnitude of $24 \mu \mathrm{m}$ sources and their associated

\footnotetext{
${ }^{10}$ Following the convention of the GLIMPSE I v2.0 enhanced data products, $\mathrm{csf}=0$ implies that no sources in the Archive Catalog are within $3^{\prime \prime}$ of the source.

$11 \mathrm{http} / / /$ www.astro.wisc.edu/glimpse/GQA-master.pdf
} 

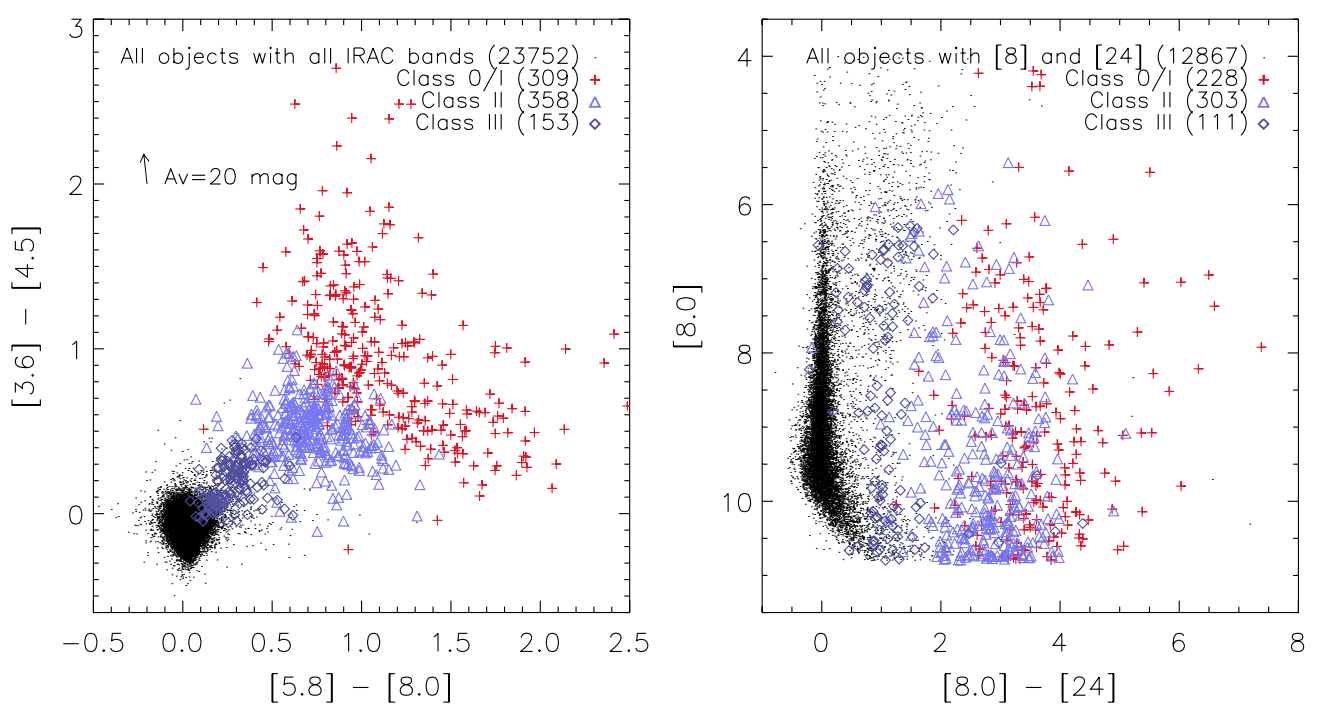

Figure 5. Left panel: IRAC color-color diagram of point sources found in the Vulpecula region. Stars with photospheric emission are marked as black dots in the figure, they have null colors in IRAC bands so they aggregate around point $(0,0)$. Color- and symbol-coded YSO candidates protrude from the bulk of the stellar sources toward the positive color quadrant due to their IR-excess emission. The reddening vector is derived from the extinction law of Flaherty (2007). Right panel: color-magnitude diagram of point sources based on MIPS and IRAC bands.

(A color version of this figure is available in the online journal.)

Table 1

Zero Points used for Flux-to-Magnitude Conversions

\begin{tabular}{|c|c|c|c|c|c|c|c|c|c|}
\hline \multirow[t]{2}{*}{ Band } & \multicolumn{3}{|c|}{ 2MASS } & \multicolumn{4}{|c|}{ IRAC } & \multicolumn{2}{|c|}{ MIPS } \\
\hline & $J$ & $H$ & $K$ & [3.6] & [4.5] & {$[5.8]$} & {$[8.0]$} & [24] & [70] \\
\hline Zero point (Jy) & 1594 & 1024 & 666.7 & 280.9 & 179.7 & 115.0 & 64.13 & 7.17 & 0.778 \\
\hline
\end{tabular}

Note. 2MASS zero points are from Cohen et al. (2003), IRAC from Reach et al. (2005), and MIPS from Rieke et al. (2008).

errors be constrained. These constraints, however, are applied at a later stage of the selection procedure (see Section 4.2).

Out of nearly $3.3 \times 10^{5}$ sources present in the GLIMPSE catalog within our search area, only $\sim 1.47 \times 10^{5}$ bypassed the initial magnitude cuts. Most of the exclusions were due to noisy detections in bands 3 and 4 of IRAC.

In the end, the initial catalog from which we search for YSO candidates contains point sources with well measured photometry and with at least one detection in IRAC bands, plus the associated 2MASS and MIPSGAL $24 \mu \mathrm{m}$ fluxes if they exist.

\subsection{Mid-IR Selection of YSO Candidates}

We start our search for YSO candidates in the initial catalog by removing extragalactic contaminants that might be misidentified as YSOs. Using the selection criteria presented in the Appendices of Gutermuth et al. (2008), which are based on a complementary analysis of the Bootes field IRAC data (Stern et al. 2005), we identify and reject six sources dominated by polycyclic aromatic hydrocarbons (PAHs) feature emission, likely star-forming galaxies or weak-line active galactic nuclei (AGNs). No sources matching the colors and magnitudes criteria for broad-line AGNs were found. Considering a search area of $6.5 \mathrm{deg}^{2}$, we find a density of extragalactic sources in Vulpecula slightly lower than 1 per $\mathrm{deg}^{2}$. This is comparable to the density of $\sim 0.5$ galaxy per $\mathrm{deg}^{2}$ found in the zone of avoidance by Marleau et al. (2008) using GLIMPSE and MIPSGAL data. We further filter the catalog according to Gutermuth et al. criteria and reject eight sources that have a large $4.5 \mu \mathrm{m}$ excess emission consistent with molecular hydrogen line emission found in regions where high-velocity outflows interact with the cold molecular cloud. The remaining sources are presumably of stellar origin.

All sources with the following color constraints are considered likely YSOs:

$$
\begin{gathered}
{[4.5]-[8.0]>0.5,} \\
{[3.6]-[5.8]>0.35} \\
{[3.6]-[5.8] \leqslant 3.5 \times([4.5]-[8.0])-1.25}
\end{gathered}
$$

Out of the $\sim 2.38 \times 10^{4}$ sources possessing all four IRAC magnitudes, we identified 820 YSO candidates based on their IRexcess emission. We further classify the selected objects according to their infrared spectral index $\alpha_{\mathrm{IR}}=\partial \log \left(\lambda F_{\lambda}\right) / \partial \log (\lambda)$ as defined by Lada (1987). We specifically compute the spectral index $\alpha_{\text {IRAC }}$ as the slope of the spectral energy distribution (SED) measured from 3.6 to $8.0 \mu \mathrm{m}$. Objects with $\alpha_{\mathrm{IRAC}}>-0.3$ have a flatish or rising SED indicating the presence of a cold dusty envelop infalling onto a central protostar; these are designated class 0/I YSOs. Objects with $-0.3>\alpha_{\text {IRAC }}>-1.6$ are classified as class II YSOs; these are pre-main-sequence stars with warm optically thick dusty disks orbiting around them. We define class III objects as having $-1.6>\alpha_{\text {IRAC }}>-2.56$ : these stars have cleared most of their circumstellar environment; their near-IR emission is mostly photospheric but they may present some excess emission above $20 \mu \mathrm{m}$. Such objects are dubbed anemic disks by Lada et al. (2006). Finally, objects with $\alpha_{\text {IRAC }}<-2.56$ are stars with photospheric emission only; this spectral index corresponds to the slope of the stellar photosphere SED in the Rayleigh-Jeans domain. 
Table 2

List of YSO Candidates and their 2MASS, IRAC, and MIPS Photometry

\begin{tabular}{|c|c|c|c|c|c|c|c|c|c|c|c|c|}
\hline \multirow[t]{2}{*}{ GLIMPSE Source Name } & \multicolumn{2}{|c|}{ Galactic } & \multicolumn{3}{|c|}{ 2MASS } & \multicolumn{4}{|c|}{ IRAC } & \multicolumn{2}{|c|}{ MIPS } & \multirow[t]{2}{*}{ Class } \\
\hline & Glon & Glat & $J$ & $H$ & $K s$ & {$[3.6]$} & {$[4.5]$} & {$[5.8]$} & {$[8.0]$} & {$[24]$} & {$[70]$} & \\
\hline SSTGLMC G060.3261-00.6407 & 60.3261 & -0.6407 & $\ldots$ & $\ldots$ & $\ldots$ & 8.68 & 8.19 & 7.74 & 7.07 & 4.55 & $\ldots$ & II \\
\hline SSTGLMC G060.1815-00.5632 & 60.1816 & -0.5632 & 12.71 & 10.61 & 9.45 & 8.31 & 8.22 & 7.89 & 7.42 & 7.16 & $\ldots$ & III \\
\hline SSTGLMC G060.3304-00.6042 & 60.3304 & -0.6042 & $\ldots$ & $\ldots$ & $\ldots$ & 12.99 & 11.40 & 10.71 & 10.14 & 4.78 & -5.65 & $0 / \mathrm{I}$ \\
\hline SSTGLMC G060.3331-00.6065 & 60.3331 & -0.6065 & 16.04 & 14.38 & 13.41 & 11.79 & 10.83 & 10.12 & 9.09 & 5.89 & & $0 / \mathrm{I}$ \\
\hline SSTGLMC G060.2117-00.6700 & 60.2118 & -0.6700 & 14.69 & 13.61 & 13.05 & 12.30 & 11.62 & 11.24 & 9.95 & $\ldots$ & & II \\
\hline SSTGLMC G060.2898-00.6427 & 60.2898 & -0.6427 & $\ldots$ & $\ldots$ & $\ldots$ & 13.14 & 12.17 & 11.44 & 10.74 & 6.98 & $\ldots$ & $0 / \mathrm{I}$ \\
\hline SSTGLMC G060.2868-00.6351 & 60.2868 & -0.6351 & $\ldots$ & $\ldots$ & $\ldots$ & 12.90 & 11.92 & 11.17 & 10.32 & 7.65 & $\ldots$ & $0 / \mathrm{I}$ \\
\hline SSTGLMC G060.1319-00.5355 & 60.1320 & -0.5355 & 10.84 & 8.99 & 8.01 & 7.30 & 7.24 & 6.94 & 6.70 & 5.44 & .. & III \\
\hline SSTGLMC G060.2180-00.6362 & 60.2180 & -0.6362 & 10.68 & 8.79 & 7.86 & 7.24 & 7.28 & 6.85 & 6.55 & 5.27 & . & III \\
\hline SSTGLMC G060.1975-00.6052 & 60.1975 & -0.6052 & $\ldots$ & $\ldots$ & $\ldots$ & 13.63 & 12.32 & 11.28 & 10.38 & 7.24 & $\ldots$ & $0 / \mathrm{I}$ \\
\hline
\end{tabular}

Note. Galactic coordinates are in units of degrees $\left({ }^{\circ}\right)$.

(This table is available in its entirety in a machine-readable form in the online journal. A portion is shown here for guidance regarding its form and content.)

Note that this YSO classification is based on the classification scheme proposed by Greene et al. (1994), except that the Flat Class $(0.3>\alpha>-0.3)$ is included into the class $0 /$ I which represents the population of protostars with infalling envelopes. Calvet et al. (1994) indeed showed that Flat Class YSOs could be interpreted as infalling envelopes. Figure 5 presents a color-color diagram based on IRAC data only; the different symbols indicate the classes associated with each YSO candidate identified in our analysis.

The next step is to exploit the extra information contained at longer wavelengths to confirm, or reclassify, the YSO candidates identified solely from their IRAC colors. We first check that the SEDs of all YSO candidates continue to rise from 8 to $24 \mu \mathrm{m}$. We also look for objects previously classified as photospheric sources that exhibit bright $24 \mu \mathrm{m}$ fluxes ([5.8] $-[24]>1.5$ ). These sources are thought to be transition disks, i.e., class II YSOs with significant dust clearing from their inner disks (Calvet et al. 2002; D'Alessio et al. 2005), these objects are of particular importance for understanding the evolution of circumstellar disks around young stars. We also check for protostar misclassifications due to extreme visual extinction levels. Gutermuth et al. (2008) suggest that if a protostar that has MIPS detection does not meet the criterion [5.8] - [24] > 4 (if they possess [5.8] photometry) or [4.5] - [24] $>4$, then it is likely a highly reddened class II YSO. Finally, we require that any sources lacking detections in some IRAC bands yet having bright $24 \mu \mathrm{m}$ fluxes ([24] $>7$ and [X] $-[24]>4.5 \mathrm{mag}$, where $[X]$ is the photometry for any IRAC detection available in our catalog) have to be added to the list of likely YSOs, and to be classified as highly embedded protostars. We apply the $24 \mu \mathrm{m}$ based color constraints on YSO candidates only if their MIPS detection reliability is $>95 \%$ (S. Shenoy 2009, private communication); thus we require that, for the MIPS photometry to be relevant for the YSO selection, the $24 \mu \mathrm{m}$ magnitude is $[24]<8.6 \mathrm{mag}$ and that its associated error is $\sigma_{[24]}<0.2 \mathrm{mag}$.

We tested the ability of the method at finding genuine YSOs on a well studied star-forming region, RCW79 (Zavagno et al. 2006), and it proved to be adequately reliable and efficient (A. Zavagno 2009, private communication). Furthermore, this method has been successfully applied to study embedded stellar clusters in NGC 1333 (Gutermuth et al. 2008), the star formation activity in the H II region W5 (Koenig et al. 2008) or in the giant molecular cloud G216-2.5 (Megeath et al. 2009). Note that other methods, also based on Spitzer colors and magnitudes, have been developed to find YSO candidates (e.g., Hartmann et al. 2005; Harvey et al. 2007; Robitaille et al. 2008; Chavarría et al. 2008).

\subsection{Final Census of YSO Candidates}

We finally find 856 YSO candidates in VulOB1: 239 are likely protostars with infalling envelops (class 0/I), 464 are disk-bearing stars (class II), and 153 are class III objects with very little circumstellar material. Among the class I objects, 15 YSO candidates are likely deeply embedded protostars, and 89 have a spectral index consistent with the Flat Class. Among the class II objects, 85 are highly reddened class II YSOs that were misclassified as protostars based on IRAC criteria only. Further 21 sources are classified as likely transition disk class II objects.

Except for a few cases, all YSO candidates have detections in all four IRAC bands, about half of them have 2MASS detections in all three $J H K$ bands, $75 \%$ have $24 \mu \mathrm{m}$ counterparts (it reaches $100 \%$ for class $0 / \mathrm{I}$ objects) and $8 \%$ have $70 \mu \mathrm{m}$ detections. Table 2 gives the coordinates and fluxes of all YSO candidates. Table 5 in the Appendix gives the coordinates, fluxes, and type of the contaminants identified in Vul OB1.

To test the robustness of our analysis relative to the interstellar extinction, we have used the visual extinction map of Vul OB1 and the formulae provided by Flaherty (2007) to deredden IRAC fluxes. We run the exact same procedure as described above, and we find that the YSO census is only marginally different from the uncorrected case (except for the class III population that is reduced by $35 \%$ ). This justifies the choice of [5.8] - [8.0] as an extinction-free indicator for finding YSOs.

\subsection{Reliability and Completeness}

In the present study, we make the deliberate choice to favor the highest possible detection reliability, at the expense of a modest completeness figure. This choice was largely motivated by the need to automate the search for YSOs over such a large area of the sky.

As mentioned in Section 4.1, we achieve a detection reliability better than $98 \%$ for the set of IRAC magnitude cuts chosen for populating our initial catalog. However, the actual detection reliability depends to a certain extent on the level of the background diffuse emission. An adequate assessment of the completeness of our YSOs catalog would require to carry out a large spectroscopic survey of the red objects in VulOB1 in order to identify genuine YSOs, and then compare the results to 


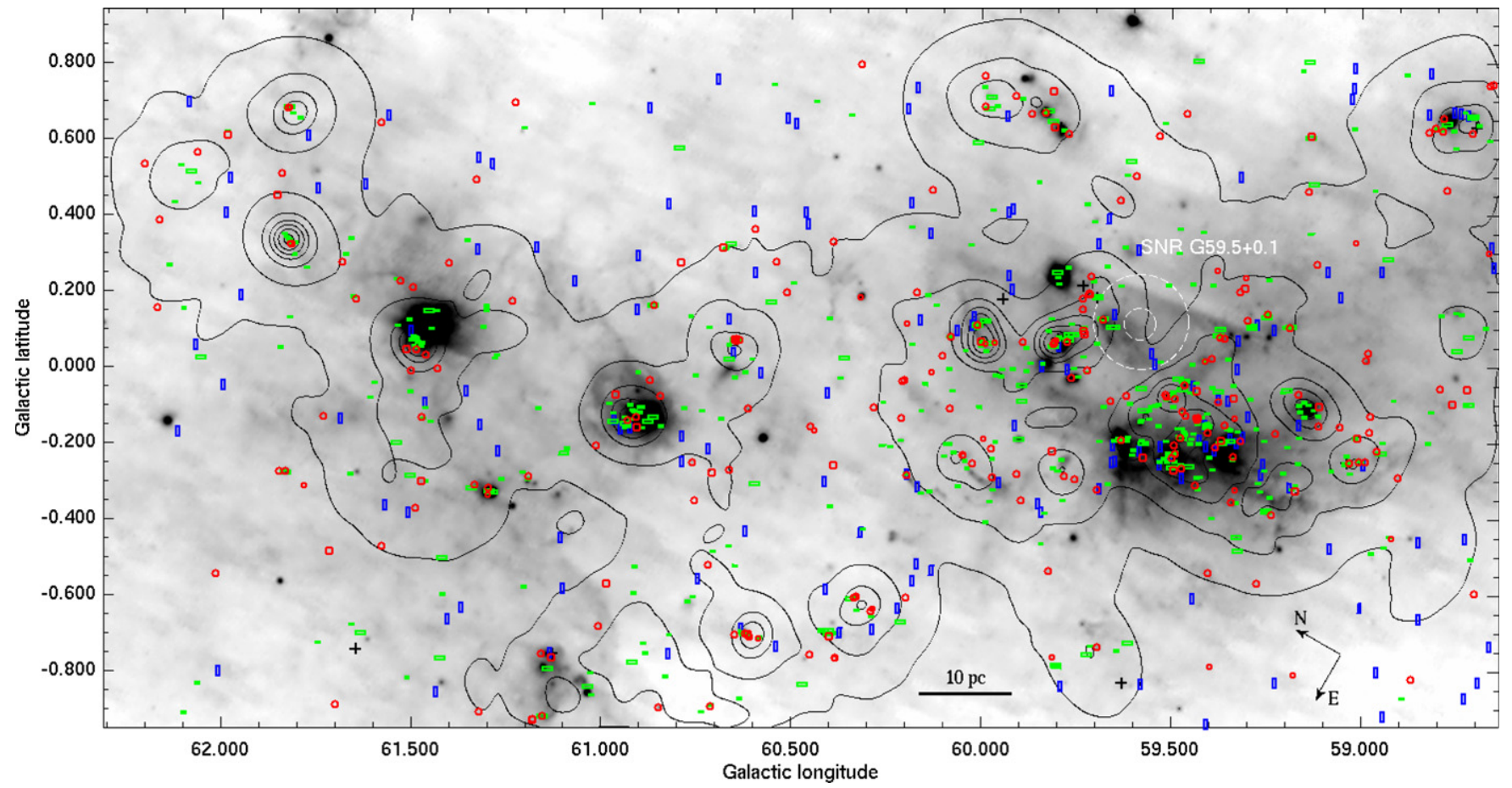

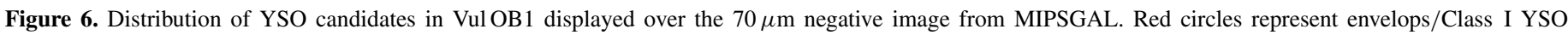

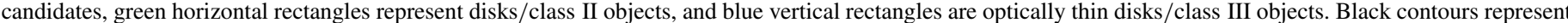

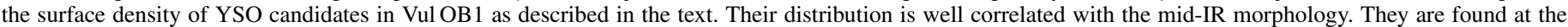

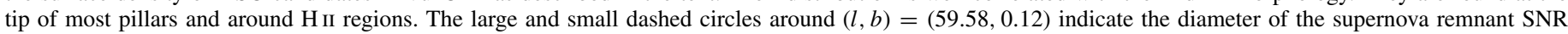
G59.5+0.1 as published in Taylor et al. (1992) and Green (2006), respectively. The orientation and scale of the image are also indicated.

our catalog. This is impractical though, given the large number of (faint) objects in Vul OB1.

Considering the stringent criteria used to reach a reliability over $98 \%$, we expect a noticeable number of YSOs to be excluded from our initial PSC. For instance, any saturated sources, that are either bright sources on faint background or faint sources on bright background, are not considered in our analysis. There are very few saturated data in IRAC images so that the YSOs selection is basically unaffected by this effect. Still, we expect that a fraction of YSO candidates will not have $24 \mu \mathrm{m}$ counterparts because of saturation issues (especially around Sh2-88B and Sh2-87 where the background is high). Extended sources are also excluded based on the selection procedure used in this work (see Section 4), but their actual number is expected to be fairly small at 2.3 kpc. Point sources with too large a magnitude error are not considered either. We set the limit for bad photometry to be $\sigma_{\text {mag }}>0.2 \mathrm{mag}$. IRAC bands 3 and 4 being the noisiest bands, the selection criterion on $\sigma_{\text {mag }}$ mostly impacts [5.6] and [8.0] detections.

The completeness of our YSO catalog is also dependent on the evolutionary stage of the object. For instance, the wavelength band $2-24 \mu \mathrm{m}$ is well suited for detecting and identifying disks around young stars (class II), but longer wavelengths are more appropriate for observing early-stage objects (class $0 / \mathrm{I}$ ). We believe that our sample of IRAC-selected YSO candidates may lack a significant fraction of these protostars. For instance, we looked for a possible correlation between the spectral index $\alpha_{\text {IRAC }}$ and the visual extinction of the YSO candidates expecting to find a population of protostars with high values of $\alpha_{\text {IRAC }}$, i.e., the youngest objects, to be the most extinct/embedded objects. However, we did not find such correlation in our sample which suggests that we might be missing the most embedded phases of star formation based on IRAC and MIPSGAL $24 \mu \mathrm{m}$ data only. Similarly, the population of class III YSOs which lacks near-IR excess emission is strongly underestimated in our study, even if they are genuine YSOs possessing $\mathrm{H} \alpha$ or X-ray emission. For all these reasons, it is very difficult to assess the completeness of our YSO catalog with confidence. We use the evolutionary models of Baraffe et al. (1998) to compute the mass of a 2 Myr old star at $2.3 \mathrm{kpc}$ with no IR-excess emission having a $3.6 \mu \mathrm{m}$ magnitude of $14 \mathrm{mag}$, and we find a mass of $\sim 1 M_{\odot}$. Considering that the $8 \mu \mathrm{m}$ channel of IRAC is the less sensitive channel (limit magnitude of $10.8 \mathrm{mag}$ ), and that most YSOs have significant IR-excess emission, we estimate our YSO catalog to be complete down to a few solar masses.

Lastly, we have to account for the possible misidentification of YSO candidates with evolved stars. The circumstellar environment of planetary nebulae (PNe) or asymptotic giant branch (AGB) stars are rich in warm dust grains emitting in the nearmid-IR such that these objects occupy the same locus in colorcolor diagrams as YSOs (Hora et al. 2008; Srinivasan et al. 2009). Following the analysis of Robitaille et al. (2008), we estimate the fraction of evolved stars in our catalog of YSO candidates to be about $25 \%$, of which most are AGB stars. We will argue in Section 5.2 that AGB stars could be further excluded from the sample of YSO candidates based on clustering criteria.

\section{RESULTS AND DISCUSSION}

\subsection{Star Formation Efficiency}

We estimate the current star formation efficiency (SFE) in Vulpecula by comparing the mass of the gas reservoir, $M_{\text {cloud }}$, with the mass that has turned into stars during the last few 

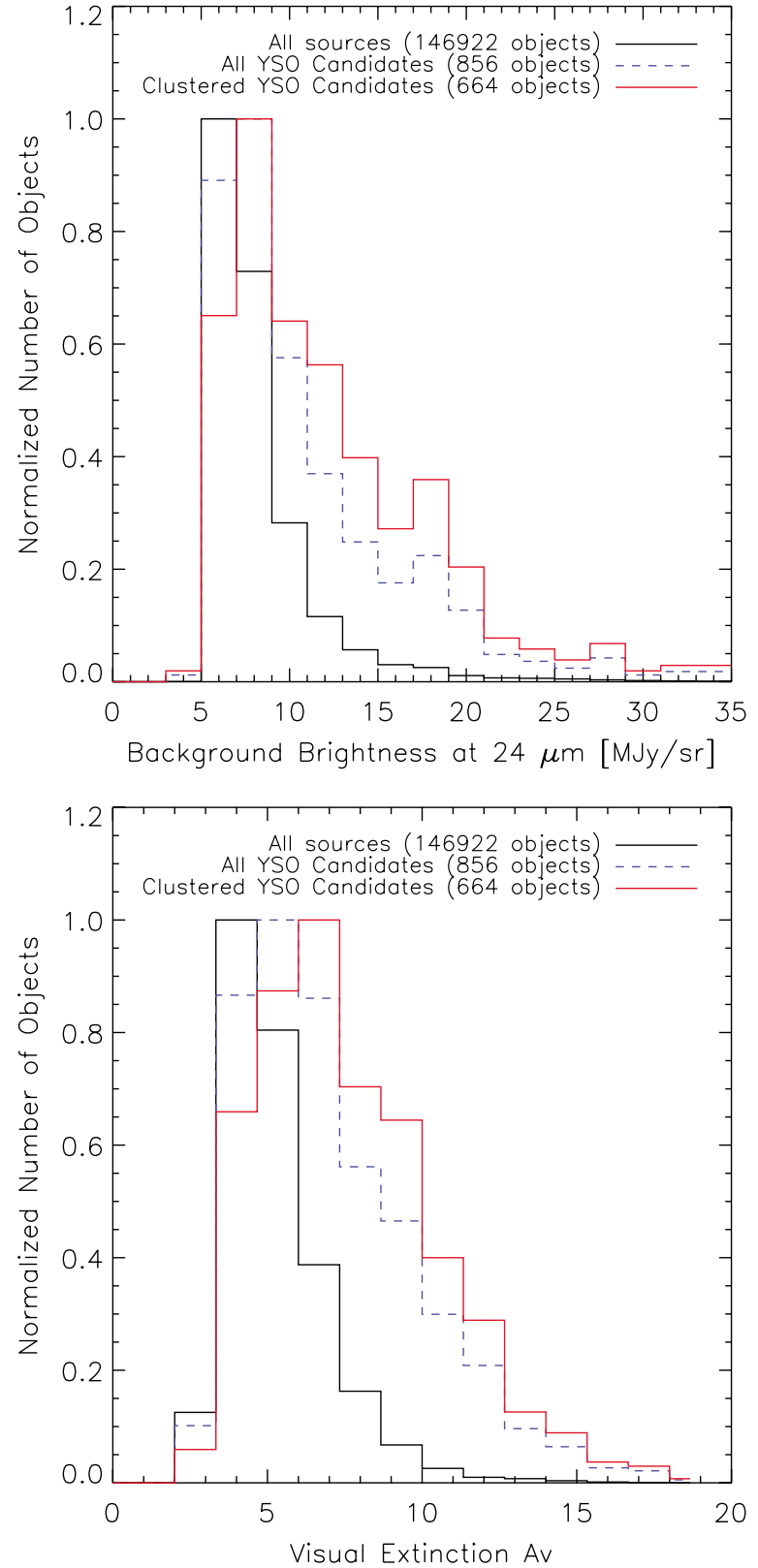

Figure 7. Histograms of $24 \mu \mathrm{m}$ background brightness (upper panel) and visual extinction (lower panel) associated with each point sources of our initial catalog. YSO candidates appear to sit preferentially on high $24 \mu \mathrm{m}$ background as well as in high extinction, i.e., embedded, regions. This is even more pronounced for clustered YSO candidates.

(A color version of this figure is available in the online journal.)

million years, $M_{\text {Yso. }}$ The SFE is then derived as follows:

$$
\mathrm{SFE}=\frac{M_{\mathrm{YSO}}}{M_{\mathrm{YSO}}+M_{\text {cloud }}} .
$$

We compute the mass of the cloud using our extinction map of Vulpecula and the formula relating the column density and the visual extinction $N_{\mathrm{H}} / A_{\mathrm{V}}=1.37 \times 10^{21} \mathrm{~cm}^{-2} \mathrm{mag}^{-1}$ assuming a value of $R_{\mathrm{V}}=5.5$ typical for molecular clouds as suggested in Evans et al. (2009). We use a contour of $A_{\mathrm{V}}=7 \mathrm{mag}$ to delineate the active star-forming region on the $A_{\mathrm{V}}$ map of Vulpecula. We find an average mass surface density of $16.2 M_{\odot} \mathrm{pc}^{-2}$. We integrate the surface density over the $2.7 \times 10^{3} \mathrm{pc}^{2}$ enclosing the $A_{\mathrm{V}}>7$ region, and we find a cloud mass $M_{\text {cloud }}=4.5 \times 10^{4} M_{\odot}$.
Since our YSO sample represents only the high end of the initial mass function (IMF), as we estimated in Section 4.4, we need to account for the missing mass of the total YSO population in order to derive a relevant $M_{\text {Yso }}$. We use the IMF of Kroupa (2001) to compute the number of stars with masses ranging from 0.01 to $50 M_{\odot}$. We normalize the IMF assuming 510 stars have masses $>1 M_{\odot}$ within the $A_{\mathrm{V}}>7$ region, and we find that VulOB1 should contain $2 \times 10^{4}$ YSOs with a total mass of $M_{\mathrm{YSO}}=4200 M_{\odot}$. We thus obtain a SFE of $\sim 8 \%$, similar to other star-forming regions in nearby molecular clouds (Evans et al. 2009).

\subsection{Spatial Distribution of YSO Candidates}

The spatial distribution of YSO candidates in Vul OB1 is presented in Figure 6. YSO candidates are not distributed randomly in the field. We rather find several coherent structures, or groups of YSO candidates, which in most cases are reminiscent of the mid-infrared morphology of the complex. For instance, the highest densities of YSO candidates are located close to, or within the three $\mathrm{H}$ II regions. Other YSOs appear to line up along IRDCs, e.g., at $(l, b)=(59.98,0.06)$ at the pedestal of the pillar VulP5 (see Figure 4); or along bright contrasted structures of the extended $24 \mu \mathrm{m}$ emission, e.g., at $(l, b)=(61.82,0.33)$ or $(l, b)=(59.80,0.64)$. YSO candidates have also been identified at the tip of most newly discovered pillar structures (see Table 4 in the Appendix and Sections 3.2 and 5.4.2).

If we assume the IMF parameters presented in Section 5.1, we estimate that the average surface density of YSO candidates is $7.4 \mathrm{YSO} \mathrm{pc}^{-2}$. This value is consistent with the typical surface density found in other star-forming regions, e.g., $13.0 \mathrm{YSO} \mathrm{pc}^{-2}$ in Serpens (Harvey et al. 2007) or 3.3 YSO pc $^{-2}$ in Lupus (Merín et al. 2008). We build a surface density map of YSO candidates following the definition of Chavarría et al. (2008). At each point of a $5^{\prime \prime}$ grid, we compute the surface density of YSOs as

$$
\sigma=\frac{N}{\pi r_{N}^{2}}
$$

where $r_{N}$ is the distance to the $N=5$ nearest neighbor. This image is then convolved with a Gaussian of FWHM $=1^{\prime}$ to obtain a smooth contour map of the surface density. Figure 6 shows the surface density contours over a $70 \mu \mathrm{m}$ image of Vul OB1. It clearly delineates the mid-IR-bright $\mathrm{H}$ II regions, plus other groups of YSO candidates associated with fainter compact mid-IR sources, e.g., at $(l, b)=(60.60,-0.70)$. The surface density peaks at $\sim 50 \mathrm{YSO} \mathrm{pc}^{-2}$ at the position $(l, b)=(59.80,0.064)$ which is coincident with the BLAST source V30, also known as IRAS $19410+2336$. This source is a candidate hypercompact (HC) $\mathrm{H}$ II region according to Chapin et al. (2008), and about 15 of our YSO candidates appear to be grouped around it (see Section 5.4.2).

In addition to this population of clustered YSO candidates, there is a population of distributed IR-excess sources. Robitaille et al. (2008) argue that a fraction of these isolated red objects could be AGB stars mistaken for YSO candidates in our selection process (they occupy the same locus in the $\mathrm{C}-\mathrm{C}$ diagram; cf. Section 4). However, Koenig et al. (2008) argue that the isolated YSO candidates could be genuine YSOs that formed from isolated events or that were ejected ${ }^{12}$ from their

\footnotetext{
12 Assuming a relative velocity of $3 \mathrm{~km} \mathrm{~s}^{-1}$ between a young star and its parental cloud, a YSO could travel $6 \mathrm{pc}$ in less than 2 Myr. This explains how genuine YSOs can contribute to the distributed population in star-forming regions.
} 


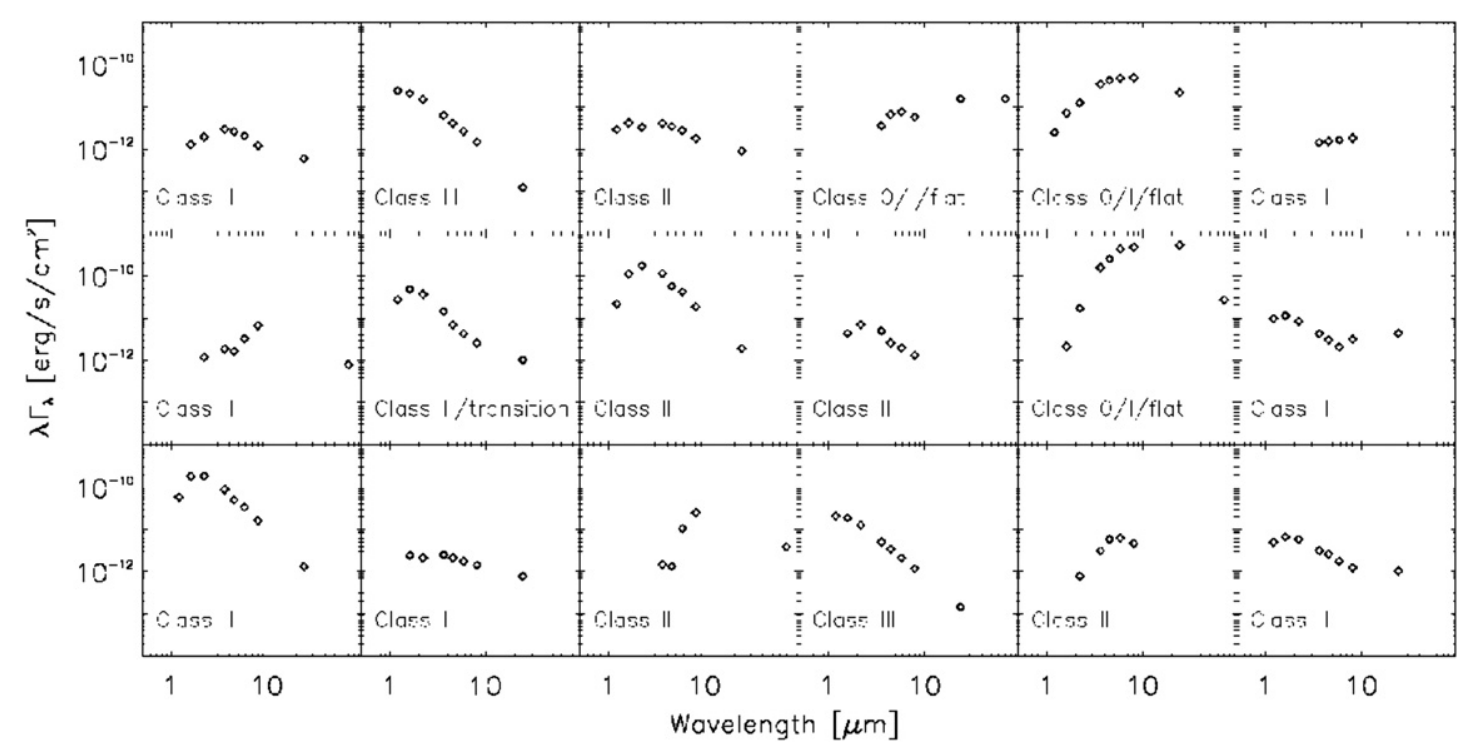

Figure 8. Sample of SEDs from our catalog of YSO candidates. Almost all YSO candidates have detection in all four IRAC bands, $75 \%$ have detections at $24 \mu \mathrm{m}, 6 \%$ at $70 \mu \mathrm{m}$, and about half in $J, H$, and $K$ bands. Our classification of each YSO candidates is shown on individual plots. The axis scale is the same for each subplot.

birthplace due to gravitational interactions with other members of their native star cluster.

We now focus our analysis on the population of clustered YSO candidates since they are more reliable tracers of triggered star formation events. Various methods can be used to discriminate between distributed/clustered populations. For instance, the two-point correlation function was used by Karr \& Martin (2003) on W5 and by Indebetouw et al. (2007) on M16, while the minimum spanning tree technique was used on W5 by Koenig et al. (2008) and on NGC 1333 by Gutermuth et al. (2008). Both methods provide valuable qualitative results but dynamical measurements are absolutely necessary to assign a definitive membership to a given YSO. Since such data are not available for Vul OB1 we opt for the simplest filtering, namely the nearest neighbor, to gain insight into the clustering properties of our YSO sample. In practice, we consider all YSO candidates and keep only those that have their nearest neighbor YSO within $3^{\prime}(2 \mathrm{pc}$ at $2.3 \mathrm{kpc})$. The result of this selection is a much more clustered distribution of YSO candidates that traces midIR morphology even more closely (192 YSO candidates are marked as isolated objects).

We also look for trends in the environment of YSO candidates compared to field stars. The top panel of Figure 7 shows that YSO candidates sit preferentially on regions of bright $24 \mu \mathrm{m}$ background emission compared to the more evolved stars. Since the $24 \mu \mathrm{m}$ emission is mostly due to the thermal emission of small dust grains excited by UV radiation, the trend for YSO candidates to sit on bright mid-IR background implies that the identified YSO candidates are largely associated with photon-dominated regions (PDRs) and H II regions. This is consistent with known scenarios of triggered star formation mechanisms and with previous studies of star-forming regions (e.g., Koenig et al. 2008). We also compare the visual extinction associated with the YSO population to the entire population of point sources found in our initial catalog (see the bottom panel of Figure 7). We find that YSO candidates are mostly located in regions of high extinction compared to field stars. This is again consistent with the idea that infant stars still live in the dense and dusty cloud from which they were born. Remarkably, the environmental trends mentioned above are even more pronounced for the population of clustered YSO candidates (see Figure 7).

Note that Chapin et al. (2008) identified a few BLAST sources in the direction of Vul OB1 that are actually located at $8.5 \mathrm{kpc}$ from the Sun (radial velocity around $-5 \mathrm{~km} \mathrm{~s}^{-1}$ ), i.e., in the Perseus arm. A dozen of our YSO candidates seem to be associated with these distant BLAST sources so that these might belong to the Perseus arm.

\subsection{Spectral Energy Distribution}

We have built SEDs for all of our YSO candidates. Figure 8 presents a small SED sample with the Class designation associated with each object.

We used the SED fitter tool developed by Robitaille et al. (2007) to extract the physical parameters of our YSO candidates. However, the limited wavelength coverage (near- to mid-IR) resulted in strong degeneracies over the output parameters, and we were unable to gain reliable information on the physical properties of our YSO candidates.

We looked for possible associations in the BLAST field to extend the wavelength coverage to the submillimeter, but in most cases several YSO candidates fell within the BLAST beam rendering the association ambiguous. For the YSO candidates that could be uniquely associated with a BLAST source, Chapin et al. (2008) provide physical parameters derived from SED fitting. They find clump masses ranging from 40 to $400 M_{\odot}$ and temperatures from 19 to $28 \mathrm{~K}$. We also use Molinari et al. (2008a) diagnostic diagrams based on [24 - 70] and [250 500] colors to identify the most massive YSOs in Vul OB1. Molinari et al. show that the high-mass analogs of the low- or intermediate-mass class 0 objects have distinctive MIPS colors. From our sample of YSO candidates, we find six objects with $[24-70]>3$, which is indicative of an SED peaking longward of $70 \mu \mathrm{m}$ presumably due to a massive infalling envelop. Three of these red MIPS sources are located next to the brightest BLAST source V30 and are barely discernible on the map, two have no BLAST counterparts, and one might have a BLAST counterpart but the association is ambiguous as another YSO candidate falls within the BLAST beam. 

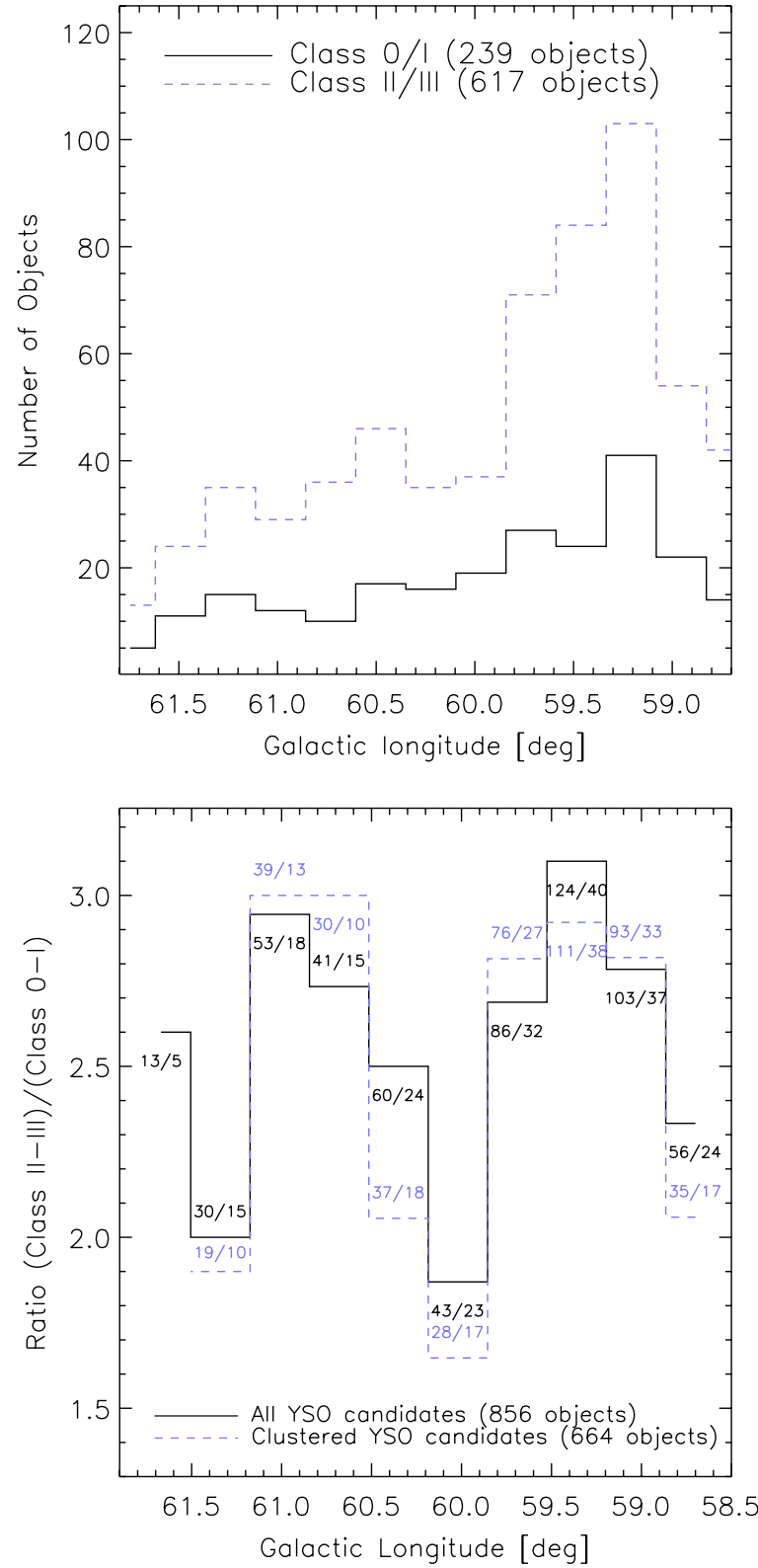

Figure 9. Top panel: distribution of class $0 / \mathrm{I}$ and II/III candidates as a function of Galactic longitude (bin size $\sim 15^{\prime}$ ). The number of YSOs increases towards lower longitudes. Bottom panel: evolution of the class II/III to class 0/I ratio as a function of longitude. The number of class $0 / \mathrm{I}$ and II/III in each bin is indicated on the figure.

(A color version of this figure is available in the online journal.)

Finally, we searched the IRSA for Bolocam sources, and we found 24 sources in VulOB1. Among them we find isolated sources not associated with any YSO candidates, these could be YSOs in their earliest evolutionary phases, i.e., starless cores emitting in the millimeter only. The other Bolocam sources are usually located close to the peaks of the YSO surface density and are associated with groups of YSO candidates. Note that almost all Bolocam sources have BLAST counterparts over the Vulpecula BLAST field.

\subsection{Cases of Triggered Star Formation? \\ 5.4.1. The Case of SNR G59.5+0.1}

Shock waves generated by supernovae can potentially trigger episodes of star formation at the interface between the SNR and the ISM (Melioli et al. 2006). The supernova remnant SNR G59.5+0.1 was first detected by Taylor et al. (1992) in the direction of Vul OB1 at the position $(l, b)=(59.58,0.12)$. Taylor et al. describe this object as a shell-type SNR with nonthermal spectral index $(\alpha<-0.4)$ and a diameter of $15^{\prime}$. They also mention the close proximity and possible association of the SNR with the HII region Sh2-86. We investigate the possibility of such an association and potential signs of triggered star formation.

Guseinov et al. (2003) computed the distance to SNR G59.5+0.1 using an empirical formula that relates the surface brightness of a SNR $(\Sigma)$ with its diameter $(D)$. They calibrated the $\Sigma-D$ relation against SNRs of known distances, and they found that SNR G59.5+0.1 lies at $\sim 11 \mathrm{kpc}$ from the Sun. This would imply that SNR G59.5+0.1 is not associated with the H II region Sh2-86. We stress however that Guseinov et al. computed the distance to G59.5+0.1 based on data from the SNR catalog of Green (2006) $\left(\Sigma_{1 \mathrm{GHz}}=18.1 \times 10^{-21} \mathrm{~W} \mathrm{~m}^{-2} \mathrm{~Hz}^{-1} \mathrm{sr}^{-1}\right.$ and $D=5^{\prime}$ from observations at $1720 \mathrm{MHz}$ ), whereas Taylor et al. report different values from observations at 327 and $4850 \mathrm{MHz}$ $\left(\Sigma_{1 \mathrm{GHz}}=0.7 \times 10^{-21} \mathrm{~W} \mathrm{~m}^{-2} \mathrm{~Hz}^{-1} \mathrm{sr}^{-1}\right.$ and $\left.D=15^{\prime}\right)$. We use the VGPS $21 \mathrm{~cm}$ data to look for traces of the SNR and settle this discrepancy. We find a circular structure centered at the position of the SNR with a diameter of $15^{\prime}$, which confirms the value found by Taylor et al. Therefore, we compute the distance to SNR G59.5+0.1 using Guseinov et al.'s formula with Taylor et al.'s measurements instead of Green's, and we find that the SNR likely lies between $2.1 \mathrm{kpc}$ and $5.3 \mathrm{kpc}$ from the Sun provided the given uncertainties of the $\Sigma-D$ relation. This distance estimate reconciles the hypothesis that SNR G59.5+0.1 is indeed associated with Sh2-86.

Reach et al. (2006) have searched for infrared counterparts to the known SNRs in the inner galactic plane using IRAC observations from the GLIMPSE survey; however, they did not detect SNR G59.5+0.1 because of the high confusion level in the vicinity of Sh2-86 structured extended emission. For the same reason, we could not detect the SNR on MIPSGAL $24 \mu \mathrm{m}$ images either. No morphological clues were found in the ancillary data mentioned in Section 2.2. Nevertheless, the distribution of YSO candidates around SNR G59.5+0.1 reveals two groups of YSOs apparently lining up along two arcs at the northern and southern rims of SNR G59.5+0.1 (cf. Figure 6). Assuming that the actual diameter of the SNR is indeed $15^{\prime}$, the two groups do not lie exactly on the rim of the shell but slightly outside. We still believe that these YSO overdensities could have arisen from the interaction of the expanding shell of SNR G59.5+0.1 with the neutral gas of Sh2-86. According to Xu et al. (2005), the age of a shell-type SNR such as SNR G59.5+0.1 with a diameter of $10 \mathrm{pc}$ (assuming a $15^{\prime}$ diameter and a distance of $2.3 \mathrm{kpc}$ ) ranges from $10^{3}$ to $10^{4}$ years old. This timescale is comparable to the lifetime of the first phases of star formation, making the SNR a possible trigger for the surrounding YSO candidates. These clues tend to confirm the association of SNR G59.5+0.1 with Sh2-86, but since we could not find unequivocal evidences for the SNR-HII region association, a fortuitous spatial coincidence cannot be excluded.

\subsubsection{Photoevaporation at the Tip of the Pillars}

An interstellar cloud exposed to the ionizing radiation of a newly formed star is compressed by an ionization-shock front which can focus the neutral gas into a compact globule. This mechanism is called radiation-driven implosion (RDI) and is described in Bertoldi (1989). In some cases, this leads to the 
birth of a second generation of stars located at the interface of the ionized and neutral gas (e.g., Reach et al. 2009).

Even though we could not identify the sculptor of the pillars described in Section 3.2, we expect the RDI mechanism to be at work in these objects. Figure 4 shows the location of our YSO candidates in pillars VulPl to VulP6 and VulP11. We find at least one YSO candidate at the tip of most of the pillars (see Figure 3 and the last column of Table 4). YSO candidates can also be found along the pillars when the gas is protruding and exposed to the ionizing star (e.g., YSO on the west side of VulP3 in Figure 4). BLAST also detected sources at the tip of every pillar found within the Vulpecula BLAST field (e.g., the bottom panel of Figure 4).

\subsubsection{Embedded Star Cluster}

Cr 404 is a young (9 Myr old; Bica et al. 2008) star cluster located at $(l, b)=(59.14,-0.11)$ embedded in Sh2-86. We find a high surface density of YSO candidates associated with this cluster. Eleven class II-III and three class I YSO candidates surround the mid-IR peak emission of $\mathrm{Cr} 404$. BLAST observations further reveal that the embedded cluster is coincident with the bright submillimeter source V18, also identified as IRAS 19403+2258. Chapin et al. (2008) classify this source as a candidate $\mathrm{HC} \mathrm{H}$ II region, i.e., the precursor of a high-mass star, and they derived a mass of $\sim 150 M_{\odot}$ and a temperature of $\sim 30 \mathrm{~K}$ for this object. The simultaneous presence of a $\mathrm{HC} \mathrm{H}$ II region ( $\sim 10^{5}$ yr lifetime) and class II YSOs (few $10^{6} \mathrm{yr}$ lifetime) around a young star cluster such as $\mathrm{Cr} 404$ is consistent with the results of Molinari (2008b) which suggests that the most massive objects in a cluster are the last ones to form.

Other cases of HC HII regions associated with groups of Spitzer-selected YSO candidates are found in the BLAST field. For instance, the BLAST source V30, the brightest and most massive clump according to Chapin et al. (2008) is coincident with the highest density of YSO candidates in the whole 6.6 $\operatorname{deg}^{2}$ map at $(l, b)=(59.80,0.064)$.

\subsubsection{Large-scale Propagating Star Formation}

Based on radio observations of $\mathrm{HI}$ supershells and on energetics arguments, Ehlerová et al. (2001) estimate that the Hi shell GS061+00+51, which encompasses Sh2-87 and Sh2-88, is 6-7 times larger and 3-4 Myr older than the shell GS59.7-0.4+44 that hosts Sh2-86. Ehlerová et al. attribute these age and size differences to the delayed expansion of $\mathrm{H}$ II regions, and they suggest that star formation might be propagating from Sh2-88 to Sh2-86. Previously, Turner (1986) suggested that the morphology of the three H II regions was shaped by a single supernova shock wave associated with the fossil HiI region Lynds $792(l, b)=(60.81,2.24)$. Since Lynds 792 is approximately equidistant from the three H II regions, Turner's scenario implies that star formation would be triggered simultaneously, as opposed to sequentially in the case of Ehlerová et al. We now test the validity of these scenarios using the supplemental information gathered in this work.

Figure 9 shows the evolution of the number of YSO candidates as a function of their Galactic longitude. The increasing number of YSO candidates toward Sh2-86 seems to favor the scenario of Ehlerová et al. since the youngest star-forming region should indeed possess the largest number of YSOs, assuming identical IMFs and instantaneous star formation. Nevertheless, the shape of this histogram could also be interpreted as Sh2-86 being a more active star-forming region than the other two Sharpless objects, creating more YSOs from a larger molecular reservoir, which is unrelated to any process of propagating star formation. For a proper interpretation of the Figure 9 histogram, we need to compare the typical lifetime of a YSO and the timescale necessary for star formation to propagate in a molecular cloud.

Nomura \& Kamaya (2001) have studied self-propagating star formation using numerical simulations and found that the time delay of sequential star formation sites against the original one, $\Delta t$, correlates with their physical separation, $\Delta x$, as $\Delta t \sim 50 \operatorname{Myr}[\Delta x /(0.5 \mathrm{kpc})]^{0.5}$. Efremov \& Elmegreen (1998) have derived a similar expression based on observations of star clusters in the Large Magellanic Cloud $(\Delta t \sim$ $\left.26 \operatorname{Myr}[\Delta x /(0.5 \mathrm{kpc})]^{0.4}\right)$. Assuming Sh2-88 and Sh2-86 are separated by $\sim 80 \mathrm{pc}$, we find that star formation would take 10-20 Myr to propagate across Vul OB1. Besides, Evans et al. (2009) estimate the lifetime of a YSO to be of the order of 13 Myr based on a large statistical sample of YSOs identified as part of the c2d legacy survey. ${ }^{13}$ If star formation was indeed propagating along the Galactic plane at the pace derived above, YSOs would only be found in a narrow slab of longitudes since they would age, and fade away in the IR, faster than the triggering shock needs to cross the molecular complex. We can already rule out this scenario based on the presence of YSOs in all three HII regions of Vul OB1 (see Figure 6). It is possible however that star formation is propagating faster than previously estimated due to the pronounced inhomogeneities of the propagation medium (cf. distribution of $\mathrm{CO}$ in Figure 1). If the propagation timescale was of the same order of the YSOs lifetime, then YSOs would be found all across Vul OB1 with a gradient in the evolutionary phases of the YSO population as a function of longitude.

We use the ratio of class II-III to class $0-$ I as an indicator of the aging of the YSO population. Figure 9 shows that this ratio does not present a gradient with respect to longitudes. This indicates that if star formation was once triggered in Vul OB1, then the triggering agent would have been independent of Galactic longitude, and this definitely rules out the suggestion of Ehlerová et al. Nonetheless, the histogram of Figure 9 is still consistent with Turner's scenario in which star formation occurs as an instantaneous burst in the three $\mathrm{H}$ II regions.

\section{CONCLUSIONS}

We have presented a thorough description of the Vulpecula OB association. We have complemented the existing observations of isolated objects in VulOB1 with a global view of the whole star-forming complex from an infrared perspective. We exploited Spitzer legacy surveys MIPSGAL and GLIMPSE data to identify 856 YSOs with IR-excess emission. We rely on the nature and properties of these objects to highlight the recent activity of star formation in the complex, and we look for evidences of triggered star formation.

We find two populations of YSO candidates: one population of distributed objects that likely contains IR-bright evolved stars and some genuine YSOs born in isolation or ejected from their parental cloud; and another population of clustered YSO candidates whose spatial distribution correlates very well with the mid-IR morphology of the complex. YSO candidates surface density peaks locally around the three Sharpless objects, the HC H II regions, and other embedded star clusters like Cr 404. The vigorous star-forming activity around these energetic

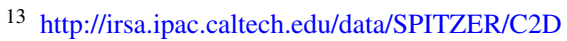




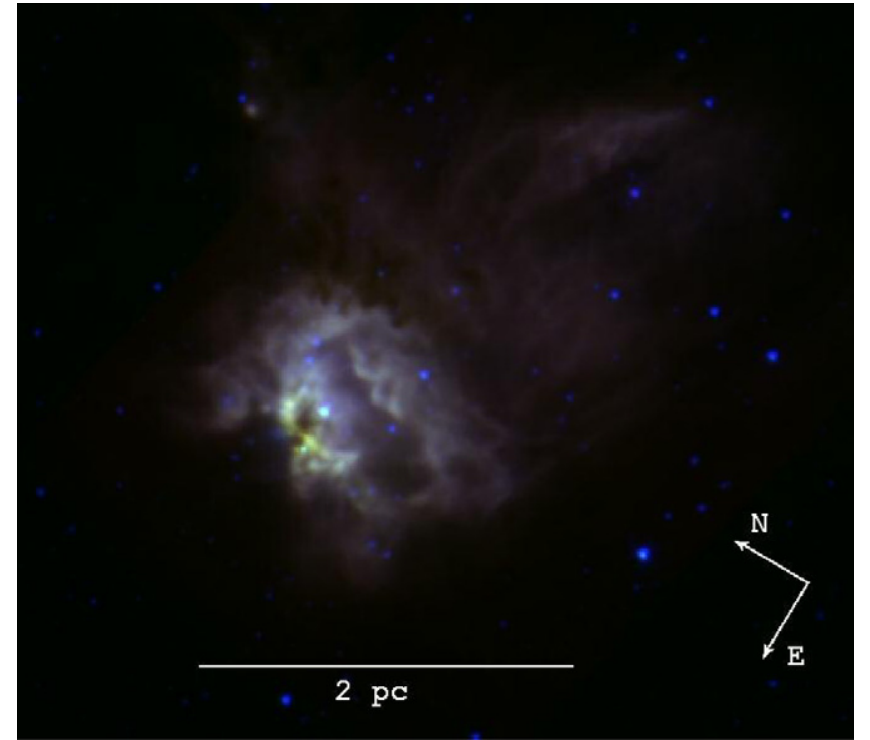

Figure 10. Composite image of the $\mathrm{H}$ II region Sh88B as seen by Spitzer (blue, green, and red are [3.6], [5.8], and [8.0], respectively).

sources is consistent with scenarios of triggered star formation mechanisms. Still, we cannot ascertain that these stars were born as a direct consequence of their extreme environment, nor that they would have formed in the absence of what we consider the triggering agents. Nevertheless, our analysis allowed to rule out the scenario of Ehlerová et al. (2001) according to which star formation was propagating from Sh2-88 to Sh2-86. We rather find that the evolutionary stage of the YSO population across Vul OB1 is homogeneous, and thus consistent with the scenario of Turner (1986).

We have further reported the discovery of a dozen pillar-like structures in VulOB1, and we comment on their morphology from the near-IR to the radio regime. We were not able to identify the energetic source(s) responsible for the molding of the pillars, but we argue that these objects are indeed associated with the OB association. Our finding of YSO candidates at the tip of most of the pillars is consistent with mechanisms of triggered star formation on small scales.

The authors thank S. Bontemps for providing the extinction map of Vul OB1. This work is based on observations made with the Spitzer Space Telescope, which is operated by the Jet Propulsion Laboratory, California Institute of Technology under a contract with NASA. This research used the facilities of the Canadian Astronomy Data Centre operated by the National Research Council of Canada with the support of the Canadian Space Agency. The National Radio Astronomy Observatory is a facility of the National Science Foundation operated under cooperative agreement by Associated Universities, Inc. The Virginia Tech Spectral-Line Survey (VTSS) is supported by the National Science Foundation. This research has made use of the SIMBAD database, operated at CDS, Strasbourg, France. This research has made use of the NASA/ IPAC Infrared Science Archive, which is operated by the Jet Propulsion Laboratory, California Institute of Technology, under contract with the National Aeronautics and Space Administration.

Facilities: Spitzer

\section{APPENDIX}

Figure 10 shows a composite image of Sh- $88 \mathrm{~B}$ in IRAC bands. Figure 11 shows a composite image of the H II region Sh86 as seen by Spitzer.

Tables 3 and 4 summarize the geometrical and morphological information extracted from the Spitzer observations for individual pillars. Table 5 gives the coordinates, fluxes, and type of the contaminants identified in Vul OB1.

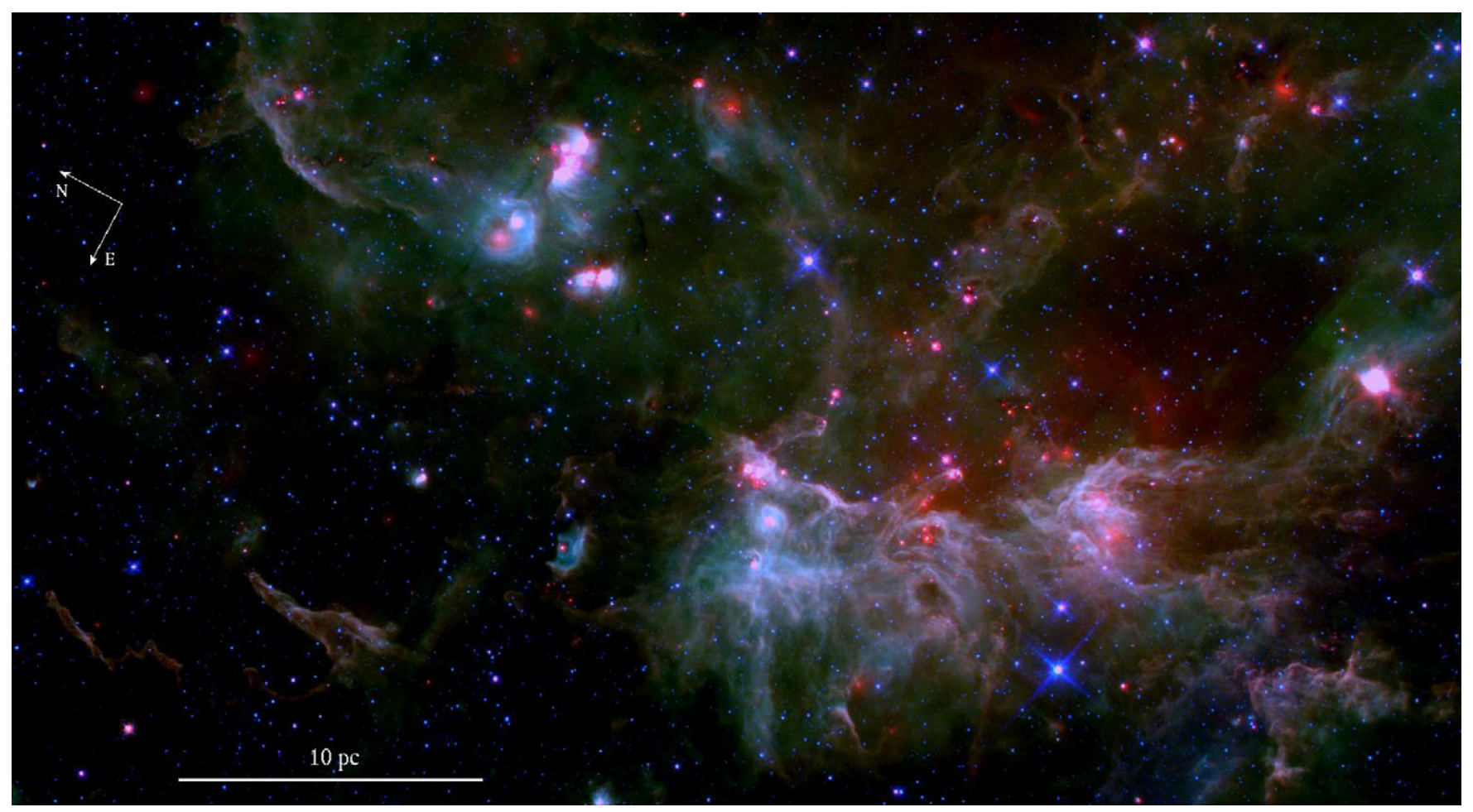

Figure 11. Composite image of the H II region Sh86 as seen by Spitzer (blue, green, and red are [5.8], [8.0], and [24], respectively). 
Table 3

Pillars Identification, Coordinates, and Approximate Size

\begin{tabular}{lccccccc}
\hline \hline Pillar ID & \multicolumn{2}{c}{ Galactic } & & \multicolumn{2}{c}{ Equatorial } & Size & Position Angle \\
\cline { 2 - 3 } & $l$ & $b$ & & R.A.(J2000) & Decl.(J2000) & & \\
\hline VulP1 & 60.18 & -0.31 & & 296.36 & 23.88 & $5-8 \times 0.5$ & 250 \\
VulP2 & 60.03 & -0.35 & & 296.32 & 23.74 & $1 \times 0.5$ & 230 \\
VulP3 & 59.97 & -0.31 & & 296.25 & 23.70 & $8 \times 2$ & 260 \\
VulP4 & 59.81 & -0.29 & & 296.15 & 23.58 & $3 \times 1$ & 268 \\
VulP5 & 60.06 & 0.10 & & 295.91 & 23.99 & $4 \times 2$ & 335 \\
VulP6 & 60.11 & 0.21 & & 295.83 & 24.10 & $5 \times 2-4$ & 350 \\
VulP7 & 60.38 & 0.21 & & 295.98 & 24.33 & $9 \times 1-4$ & 17 \\
VulP8 & 60.90 & 0.22 & & 296.26 & 24.78 & $11 \times 4$ & 55 \\
VulP9 & 61.10 & -0.45 & & 297.00 & 24.61 & $4 \times 1$ & 158 \\
VulP10 & 60.77 & -0.63 & & 297.00 & 24.24 & $4 \times 2$ & 190 \\
VulP11 & 60.21 & -0.45 & & 296.52 & 23.84 & $4 \times 2$ & 280 \\
VulP12 & 59.60 & -0.14 & & 295.90 & 23.47 & $3 \times 1$ & 133 \\
VulP13 & 59.51 & -0.22 & & 295.92 & 23.36 & $3 \times 0.5$ & 160 \\
VulP14 & 59.49 & -0.19 & & 295.88 & 23.35 & $2 \times 0.5$ & 150 \\
\hline
\end{tabular}

Notes. Coordinates and position angles are in units of degrees. Pillar sizes are in units of arcminutes. Position angles are given between the long axis of the pillar and the west axis, positive values are toward the north.

Table 4

Morphological Description of the Pillar Structures Identified in Vul OB1

\begin{tabular}{|c|c|c|c|c|c|c|c|}
\hline \multirow[t]{2}{*}{ Pillar ID } & \multicolumn{2}{|c|}{ MIPS } & \multicolumn{4}{|c|}{ IRAC } & \multirow[t]{2}{*}{$\overline{\mathrm{YSO}^{\mathrm{a}}}$} \\
\hline & [70] & {$[24]$} & {$[8.0]$} & {$[5.8]$} & [4.5] & {$[3.6]$} & \\
\hline VulPl & $\begin{array}{c}\text { Bright pillar } \\
\text { Compact source }\end{array}$ & Structured & Structured & Faint & None & Faint & $\mathrm{Y}$ \\
\hline VulP2 & Faint & Faint & Faint & None & None & None & $\mathrm{N}$ \\
\hline$\overline{V u l P 3}$ & $\begin{array}{c}\text { Bright pillar } \\
\text { Compact source }\end{array}$ & $\begin{array}{c}\text { Bright } \\
\text { Structured }\end{array}$ & $\begin{array}{c}\text { Bright } \\
\text { Structured }\end{array}$ & Faint & Faint & Faint & $\mathrm{Y}$ \\
\hline VulP4 & Faint tip & Faint & Faint & None & None & None & $\mathrm{Y}$ \\
\hline VulP5 & $\begin{array}{c}\text { Faint pillar } \\
\text { Pedestal }\end{array}$ & $\begin{array}{c}\text { Bright pillar } \\
\text { Pedestal }\end{array}$ & $\begin{array}{l}\text { Bright pillar } \\
\text { Pedestal }\end{array}$ & $\begin{array}{c}\text { No pillar } \\
\text { Faint pedestal }\end{array}$ & $\begin{array}{c}\text { No pillar } \\
\text { Faint pedestal }\end{array}$ & $\begin{array}{c}\text { No pillar } \\
\text { Faint pedestal }\end{array}$ & $\mathrm{Y}$ \\
\hline VulP6 & Faint & Faint & Faint & None & None & None & $\mathrm{Y}$ \\
\hline VulP7 & $\begin{array}{c}\text { Faint } \\
\text { Structured } \\
\end{array}$ & $\begin{array}{c}\text { Bright } \\
\text { Structured } \\
\end{array}$ & $\begin{array}{c}\text { Faint } \\
\text { Structured }\end{array}$ & None & None & None & $\mathrm{Y}$ \\
\hline VulP8 & Faint tip & $\begin{array}{c}\text { Faint } \\
\text { Structured }\end{array}$ & $\begin{array}{c}\text { Faint } \\
\text { Structured }\end{array}$ & None & None & None & $\mathrm{Y}$ \\
\hline VulP10 & Faint & $\begin{array}{c}\text { Bright tip } \\
\text { Possible jets }\end{array}$ & Faint & None & None & None & $\mathrm{Y}$ \\
\hline VulPl1 & Bright tip & Bright tip & Bright tip & Faint tip & Faint tip & Faint tip & $\mathrm{N}$ \\
\hline VulP12 & $\begin{array}{l}\text { Bright tip } \\
\text { Pedestal }\end{array}$ & $\begin{array}{l}\text { Bright tip } \\
\text { Pedestal }\end{array}$ & $\begin{array}{l}\text { Bright tip } \\
\text { Pedestal }\end{array}$ & Faint & Faint & Faint & $\mathrm{Y}$ \\
\hline VulPl3 & Faint & Bright edges & Bright edges & None & None & None & $\mathrm{Y}$ \\
\hline VulPl4 & Bright tip & Bright tip & $\begin{array}{l}\text { Bow shock } \\
\text { Bright tip }\end{array}$ & Bow shock & Bow shock & Bow shock & $\mathrm{Y}$ \\
\hline
\end{tabular}

Note. ${ }^{\mathrm{a}}$ Indicates the presence of a YSO candidate at the tip of the pillar.

Table 5

List of Point Sources Excluded from the YSO Catalog and Identified as Probable Contaminants Based on their IRAC Fluxes

\begin{tabular}{|c|c|c|c|c|c|c|c|c|c|c|c|c|}
\hline \multirow[t]{2}{*}{ GLIMPSE Source Name } & \multicolumn{2}{|c|}{ Galactic } & \multicolumn{3}{|c|}{ 2MASS } & \multicolumn{4}{|c|}{ IRAC } & \multicolumn{2}{|c|}{ MIPS } & \multirow[t]{2}{*}{ Type } \\
\hline & Glon & Glat & $J$ & $H$ & Ks & {$[3.6]$} & {$[4.5]$} & {$[5.8]$} & {$[8.0]$} & {$[24]$} & [70] & \\
\hline SSTGLMC G059.6291-00.8301 & 59.6291 & -0.8301 & 12.97 & 12.59 & 12.35 & 11.96 & 11.63 & 11.41 & 9.84 & & & Galaxy \\
\hline SSTGLMC G059.7319+00.2144 & 59.7320 & 0.2144 & 12.95 & 12.47 & 12.23 & 11.98 & 11.71 & 11.68 & 10.23 & 7.77 & & Galaxy \\
\hline SSTGLMC G059.9431+00.1783 & 59.9432 & 0.1783 & & 15.06 & 14.19 & 13.02 & 12.40 & 11.68 & 9.76 & 6.19 & & Galaxy \\
\hline SSTGLMC G060.9219-00.1041 & 60.9220 & -0.1041 & 14.37 & 13.44 & 12.98 & 12.15 & 11.87 & 11.83 & 10.53 & $\ldots$ & . & Galaxy \\
\hline SSTGLMC G061.1282-00.7549 & 61.1282 & -0.7549 & 13.60 & 12.59 & 12.32 & 12.11 & 12.14 & 11.77 & 10.63 & $\ldots$ & & Galaxy \\
\hline SSTGLMC G061.6453-00.7439 & 61.6454 & -0.7439 & $\ldots$ & $\ldots$ & $\ldots$ & 12.19 & 11.91 & 11.79 & 9.53 & $\ldots$ & $\ldots$ & Galaxy \\
\hline SSTGLMC G060.0154+00.1111 & 60.0154 & 0.1111 & $\ldots$ & $\ldots$ & .. & 12.88 & 10.83 & 10.01 & 9.56 & $\ldots$ & -4.96 & Shock \\
\hline SSTGLM & 59.6940 & 0 . & $\ldots$ & $\ldots$ & .. & 12.80 & 11.62 & 11.14 & 10.69 & $\ldots$ & -4.82 & Shock \\
\hline SST & 59.7893 & 0.6298 & $\ldots$ & 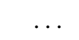 & 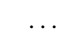 & 12.61 & 10.75 & 10.19 & 9.27 & $\ldots$ & -4.81 & Shock \\
\hline SSTGLMC G059.3780-00.2438 & 59.3781 & -0.2438 & $\ldots$ & ... & 14.84 & 12.76 & 11.23 & 10.63 & 10.30 & 3.14 & -4.77 & Shock \\
\hline SSTGLMC G059.7973+00.0750 & 59.7973 & 0.0750 & $\ldots$ & 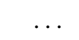 & .. & 13.22 & 11.04 & 11.11 & 10.75 & $\ldots$ & $\ldots$ & Shock \\
\hline SSTGLMC G059.6366-00.1864 & 59.6366 & -0.1864 & & & 14.73 & 11.48 & 9.56 & 8.79 & 8.33 & 3.13 & & Shock \\
\hline SSTGLMC G058.6970+00.6316 & 58.6971 & 0.6316 & & & & 12.88 & 11.41 & 10.80 & 9.70 & 3.17 & -4.15 & Shock \\
\hline SSTGLMC G059.0385-00.2512 & 59.0386 & -0.2512 & $\ldots$ & 14.47 & 13.19 & 11.32 & 10.15 & 9.89 & 8.85 & 3.67 & $\ldots$ & Shock \\
\hline
\end{tabular}

Note. Coordinates are in units of degrees $\left(^{\circ}\right)$. 


\section{REFERENCES}

Adams, F. C., Lada, C. J., \& Shu, F. H. 1987, ApJ, 312, 788

Allen, L. E., et al. 2004, ApJS, 154, 363

Aller, L. H., et al. 1982, Landolt-Bornstein Numerical Data and Functional Relations in Science and Technology, Vol. 2 (Berlin: Springer)

Anantharamaiah, K. R., Viallefond, F., Mohan, N. R., Goss, W. M., \& Zhao, J. H. 2000, ApJ, 537, 613

André, P., Motte, F., \& Neri, R. 2000, in ASP. Conf. Ser. 217, Imaging at Radio Through Submillimeter Wavelengths, ed. J. G. Mangum \& S. J. E. Radford (San Francisco, CA: ASP), 152

André, P., Ward-Thompson, D., \& Barsony, M. 1993, ApJ, 406, 122

Baraffe, I., Chabrier, G., Allard, F., \& Hauschildt, P. H. 1998, A\&A, 337, 403

Barsony, M. 1989, ApJ, 345, 268

Benjamin, R. A., et al. 2003, PASP, 115, 953

Bertoldi, F. 1989, ApJ, 346, 735

Bica, E., Bonatto, C., \& Dutra, C. M. 2008, A\&A, 489, 1129

Bonnell, I. A., Bate, M. R., \& Price, N. M. 1996, MNRAS, 279, 121

Bowler, B. P., Waller, W. H., Megeath, S. T., Patten, B. M., \& Tamura, M. 2009, AJ, 137, 3685

Brand, J., \& Blitz, L. 1993, A\&A, 275, 67

Calvet, N., D’Alessio, P., Hartmann, L., Wilner, D., Walsh, A., \& Sitko, M. 2002, ApJ, 568, 1008

Calvet, N., Hartmann, L., Kenyon, S. J., \& Whitney, B. A. 1994, ApJ, 434, 330

Cappa, C., Pineault, S., Arnal, E. M., \& Cichowolski, S. 2002, A\&A, 395, 955

Carey, S. J., et al. 2009, PASP, 121, 76

Carlqvist, P., Gahm, G. F., \& Kristen, H. 2003, A\&A, 403, 399

Chapin, E. L., et al. 2008, ApJ, 681, 428

Chavarría, L. A., Allen, L. E., Hora, J. L., Brunt, C. M., \& Fazio, G. G. 2008, ApJ, 682,445

Cohen, M., Wheaton, W. A., \& Megeath, S. T. 2003, AJ, 126, 1090

D’Alessio, P., et al. 2005, ApJ, 621, 461

Dame, T. M., Hartmann, D., \& Thaddeus, P. 2001, ApJ, 547, 792

Deharveng, L., Nadeau, D., Zavagno, A., \& Caplan, J. 2000, A\&A, 360, 1107

Dennison, B., Simonetti, J. H., \& Topasna, G. A. 1998, PASA, 15, 147

Diolaiti, E., Bendinelli, O., Bonaccini, D., Close, L., Currie, D., \& Parmeggiani, G. 2000, A\&AS, 147, 335

Efremov, Y. N., \& Elmegreen, B. G. 1998, MNRAS, 299, 588

Ehlerová, S., Palouš, J., \& Huchtmeier, W. K. 2001, A\&A, 374, 682

Elmegreen, B. G. 1998, in ASP Conf. Ser. 148, Observations and Theory of Dynamical Triggers for Star Formation, ed. C.E. Woodward, J.M. Shull, \& H.A. Thronson, Jr. (San Francisco, CA: ASP), 150

Evans, N. J., et al. 2009, ApJS, 181, 321

Fazio, G. G., et al. 2004, ApJS, 154, 10

Felli, M., \& Harten, R. H. 1981, A\&A, 100, 42

Fich, M., \& Blitz, L. 1984, ApJ, 279, 125

Flaherty, K. M. 2007, ApJ, 663, 1069

Garmany, C. D., \& Stencel, R. E. 1992, A\&AS, 94, 21

Green, D. A. 2006, A Catalogue of Galactic Supernova Remnants (2006 April version), Astrophysics Group, Cavendish Laboratory, Cambridge, UK (available at http://www.mrao.cam.ac.uk/surveys/snrs/)

Greene, T. P., Wilking, B. A., Andre, P., Young, E. T., \& Lada, C. J. 1994, ApJ, 434,614

Gritschneder, M., Naab, T., Walch, S., Burkert, A., \& Heitsch, F. 2009, ApJ, 694, 26

Guetter, H. H. 1992, AJ, 103, 197

Guieu, S., et al. 2009, ApJ, 697, 787

Guseinov, O. H., Ankay, A., Sezer, A., \& Tagieva, S. O. 2003, Astron. Astrophys. Trans., 22, 273

Gutermuth, R. A., et al. 2008, ApJ, 674, 336

Hartmann, L., Megeath, S. T., Allen, L., Luhman, K., Calvet, N., D’Alessio, P., Franco-Hernandez, R., \& Fazio, G. 2005, ApJ, 629, 881

Harvey, P., Merín, B., Huard, T. L., Rebull, L. M., Chapman, N., Evans, N. J., \& Myers, P. C. 2007, ApJ, 623, 1149

Hester, J. J., et al. 1996, AJ, 111, 2349

Hosokawa, T., \& Inutsuka, S.-i. 2006, ApJ, 648, 131

Hora, J. L., et al. 2008, AJ, 135, 726

Hoyle, F., Shanks, T., \& Tanvir, N. R. 2003, MNRAS, 345, 269

Indebetouw, R., Robitaille, T. P., Whitney, B. A., Churchwell, E., Babler, B., Meade, M., Watson, C., \& Wolfire, M. 2007, ApJ, 666, 321

Indebetouw, R., et al. 2005, ApJ, 619, 931

Johnson, H. L. 1958, Lowell Obs. Bull., 4, 37
Jørgensen, J. K., Schöier, F. L., \& van Dishoeck, E. F. 2002, A\&A, 389, 908

Karr, J. L., \& Martin, P. G. 2003, ApJ, 595, 900

Kharchenko, N. V., Piskunov, A. E., Röser, S., Schilbach, E., \& Scholz, R. D. 2005, A\&A, 438, 1163

Koenig, X. P., Allen, L. E., Gutermuth, R. A., Hora, J. L., Brunt, C. M., \& Muzerolle, J. 2008, ApJ, 688, 1142

Kroupa, P. 2001, MNRAS, 322, 231

Lada, C. J. 1987, in IAU Symp., Star-Forming Regions,115, ed. M. Peimbert \& J. Jugaku (Dordrecht: Reidel), 1

Lada, C. J., et al. 2006, AJ, 131, 1574

Lefloch, B., \& Lazareff, B. 1994, A\&A, 289, 559

Lortet-Zuckermann, M. C. 1974, A\&A, 30, 67

Lutz, D. 1999, in The Universe as Seen by ISO, ed. P. Cox \& M. F. Kessler (ESA SP-427; Noordwijk: ESA), 63

Makovoz, D., Khan, I., \& Masci, F. 2006, Proc. SPIE, 6065, 330

Marleau, F., et al. 2008, AJ, 136, 662

Massey, P., Johnson, K. E., \& Degioia-Eastwood, K. 1995, ApJ, 454, 151

McKee, C. F., \& Ostriker, E. C. 2007, ARA\&A, 45, 565

Meade, M. R., et al. 2007, GLIMPSE I v2.0 Data Release, http://data.spitzer. caltech.edu/popular/glimpse/20070416_enhanced_v2/Documents/glimpse1 dataprod_v2.0.pdf

Megeath, S. T., Allgaier, E., Young, E., Allen, T., Pipher, J. L., \& Wilson, T. L. 2009, AJ, 137, 4072

Melioli, C., de Gouveia Dal Pino, E. M., de La Reza, R., \& Raga, A. 2006, MNRAS, 373, 811

Merín, B., et al. 2008, ApJS, 177, 151

Miao, J., White, G. J., Nelson, R., Thompson, M., \& Morgan, L. 2006, MNRAS, 369,143

Mizuno, D. R., et al. 2008, PASP, 120, 1028

Mizuta, A., Kane, J. O., Pound, M. W., Remington, B. A., Ryutov, D. D., \& Takabe, H. 2006, ApJ, 647, 1151

Molinari, S. 2008, in 37th COSPAR Scientific Assembly 37, 2085

Molinari, S., Pezzuto, S., Cesaroni, R., Brand, J., Faustini, F., \& Testi, L. 2008, A\&A, 481, 345

Nomura, H., \& Kamaya, H. 2001, AJ, 121, 1024

Pascale, E., et al. 2008, ApJ, 681, 400

Pigulski, A., Kolaczkowski, Z., \& Kopacki, G. 2000, Acta Astron., 50, 113

Price, D. J., \& Bate, M. R. 2009, MNRAS, 398, 33

Reach, W. T., et al. 2004, ApJS, 154, 385

Reach, W. T., et al. 2005, PASP, 117, 978

Reach, W. T., et al. 2006, AJ, 131, 1479

Reach, W. T., et al. 2009, ApJ, 690, 683

Reed, B. C. 2003, AJ, 125, 2531

Rieke, G. H., et al. 2004, ApJS, 154, 25

Rieke, G. H., et al. 2008, AJ, 135, 2245

Robitaille, T. P., Whitney, B. A., Indebetouw, R., \& Wood, K. 2007, ApJS, 169 , 328

Robitaille, T. P., et al. 2008, AJ, 136, 2413

Rownd, B. K., \& Young, J. S. 1999, AJ, 118, 670

Schneider, N., Bontemps, S., Simon, R., Jakob, H., Motte, F., Miller, M., Kramer, C., \& Stutzki, J. 2006, A\&A, 458, 855

Sharpless, S. 1959, ApJS, 4, 257

Spitzer, L. J. 1954, ApJ, 120, 1

Srinivasan, S., et al. 2009, AJ, 137, 4810

Stern, D., et al. 2005, ApJ, 631, 163

Stetson, P. B. 1987, PASP, 99, 191

Stil, J. M., et al. 2006, AJ, 132, 1158

Stone, M. E. 1970, ApJ, 159, 293

Sugitani, K., et al. 2002, ApJ, 565, 25

Taylor, A. R., Wallace, B. J., \& Goss, W. M. 1992, AJ, 103, 931

Terebey, S., Shu, F. H., \& Cassen, P. 1984, ApJ, 286, 529

Turner, D. G. 1986, A\&A, 167, 157

Urquhart, J. S., White, G. J., Pilbratt, G. L., \& Fridlund, C. V. M. 2003, A\&A, 409, 193

Vicente, S. M., \& Alves, J. 2005, A\&A, 441, 195

Wegner, W. 2006, MNRAS, 371, 185

Werner, M. W., et al. 2004, ApJS, 154, 1

Xu, J. W., Zhang, X. Z., \& Han, J. L. 2005, Chin. J. Astron. Astrophys., 5, 165

Xue, R., \& Wu, Y. 2008, ApJ, 680, 446

Zavagno, A., Deharveng, L., Comerón, F., Bran, J., Massi, F., Caplan, J., \& Russeil, D. 2006, A\&A, 446, 171 